\title{
Residual Thermal Strain Distribution Measurement of Underfills in Flip Chip Electronic Packages by an Inverse Approach Based on the Sampling Moiré Method
}

\author{
Q. Wang ${ }^{1}$ (D) S. Ri ${ }^{1} \cdot$ T. Enomoto ${ }^{2}$
}

Received: 2 July 2019 / Accepted: 16 December 2019 / Published online: 15 January 2020

(C) The Author(s) 2020

\begin{abstract}
Residual deformation evaluation of underfill (UF) materials in flip chips is crucial to improve the reliability of electronic packages. In this study, we propose to evaluate the residual thermal strain distributions using an inverse method based on the sampling moiré technique. Even if a grid pattern is fabricated on the specimen at room temperature, the residual strain distributions at an arbitrary temperature relative to the specimen formation temperature can be successfully calculated. The residual strain distributions relative to the free contraction state at an arbitrary temperature can also be measured when the coefficient of thermal expansion is available. A thermal chamber for flip chips was designed under a laser scanning microscope. Using the proposed method, the normal, shear and principal internal strain distributions and deformation characteristics of two kinds of UFs in flip chips were investigated relative to $150{ }^{\circ} \mathrm{C}$. The strains of the UF with low glass transition temperature (UF-A) concentrate near the die material, especially at the die corner, while the strain concentration of the underfill with high glass transition temperature (UF-B) mainly occurs at the die corner and the buffer layer. The maximum principal strain of UF-A is greater than that of UF-B around the die corner. The residual maximum principal strain distributions relative to the free contraction state at $25^{\circ} \mathrm{C}$ were compared with the simulation results by the finite element method. The residual strain distribution trends from experiments are consistent with those from simulations.
\end{abstract}

Keywords Internal strain · Residual stress · Flip chip · Underfill $\cdot$ Moiré $\cdot$ Grid $\cdot$ Finite element method

\section{Introduction}

With the development of high-density semiconductor packages, to achieve high functions including heat resistance, toughness, heat dissipation, etc., more and more attention has been focused on the underfill (hereinafter referred to as UF) which is a semiconductor encapsulant resin [1]. However, due to the strong nonlinearity of the UF physical properties and the complex micro-nano compositions, optimum design of UF is quite difficult.

Q. Wang

wang.qinghua@ aist.go.jp

1 Research Institute for Measurement and Analytical Instrumentation, National Metrology Institute of Japan (NMIJ), National Institute of Advanced Industrial Science and Technology (AIST), 1-1-1 Umezono, Tsukuba, Ibaraki 305-3568, Japan

2 Technical R\&D DIV., Namics Corporation, 3993 Nigorikawa, Kita-ku, Niigata-City, Niigata 950-3131, Japan
UF is a liquid thermosetting resin and injected into a flip chip package (hereinafter referred to as FCPKG) at normal temperature, cured at high temperature, and cooled to room temperature again. Due to the difference of thermal expansion coefficients of UF, die and other materials in FCPKG, there is internal residual stress in UF and other materials. The internal stress is prone to cause a crack, leading to reliability decline of FCPKG. Since stress and strain are directly related, measurement of internal strain in FCPKG is useful for the optimal design of UF and life extension of FCPKG.

Besides residual stress, strain measurement is also essential for evaluating mechanical properties, instability behaviors, and crack initiation and propagation of materials and structures. At present, the full-field, non-contact and nondestructive deformation measurement techniques mainly include the moiré methods $[2,3]$, the digital image correlation (DIC) method [4], geometric phase analysis (GPA) [5, 6], the Fourier transform (FT) method, electronic speckle pattern interferometry (ESPI) [7]. Among these techniques, the DIC method is simple but powerless against noise because the deformation carrier is speckle, GPA and FT are weak for 
complex deformation measurement as multiple frequencies exist, and ESPI is highly sensitive to vibration.

The commonly used moire methods include the microscope scanning moiré method [8] such as electron moiré and laser moiré, moiré interferometry [9], the CCD or CMOS moiré method (abbreviated by the CCD moire method in the following), the digital/overlapped moiré method, the temporal phase-shifting moiré method and the sampling moiré method [10-12]. The first four kinds of moiré methods use the fringecentering technique, and the last two kinds of moire methods use the phase-shifting technique [13] to process moiré fringes for calculating deformation distributions.

The microscope scanning moiré method has been reported for residual strain/stress measurement of composite materials $[14,15]$, and moiré interferometry has been applied to residual strain/stress measurement of electronic packages and composite materials [16-20].

The present moiré techniques for deformation measurements mainly have three problems. 1) In the moiré methods using the fringe-centering technique, only the information of centerlines of moiré fringes is used, and thus the deformation measurement accuracy is low. Besides, it is difficult to automatically batch deformation measurement, because the centerlines of moiré fringes often need to be manually repaired. 2) Although the temporal phase-shifting technique can improve the deformation measurement accuracy, a phase-shifting device is necessary, and it takes time to record several images making this technique not suitable for dynamic analysis. 3) Since the deformation sensitivity is very high in moiré interferometry, moiré fringes are too dense to be recorded and thus cannot be analyzed in some areas with large deformation, such as the area around a die corner in FCPKG where UF sharply deforms.

Aimed at the above problems, this study concentrates on accurate measurement of internal residual strain distributions including $x$-direction, $y$-direction, shear and principal strains from a single-shot periodic pattern even in the case of large deformation. For nondestructive measurement of residual thermal strain distributions, an inverse approach based on the sampling moiré technique is proposed by combining the spatial phase-shifting sampling moiré method and an inverse idea. The residual deformation behaviors of two kinds of UFs with different glass transition temperatures in FCPKGs were investigated, and compared with the simulation results of the finite element method (FEM).

\section{Principle of Residual Thermal Strain Measurement}

\section{Sampling Moiré Generation and Phase Extraction}

The grid patterns at different temperatures can be used to generate different sampling moiré fringes digitally for deformation measurement. After using a low pass filter or FT, a cross grid can be separated into two parallel gratings, i.e., grating $X$ and grating $Y$ (Fig. 1). At room temperature $T r$ when the specimen grid is fabricated, suppose the pitch of grating $X$ in the $x$ direction is $P_{x}$, and the pitch of grating $Y$ in the $y$ direction is $P_{y}$ respectively. Since the phase analysis principles in the $x$ and $y$ directions are the same, the moiré generation, as well as the phase extraction process, will be introduced taking grating $Y$ as an example. The intensity of grating $Y$ at room temperature can be written as

$I_{y}=A_{y} \cos \left(2 \pi \frac{y}{P_{y}}\right)+B_{y}=A_{y} \cos \varphi_{y}+B_{y}$

where $A_{y}$ means the modulated amplitude, $B_{y}$ gives the background and high-order intensities, and $\varphi_{y}$ stands for the phase of grating $Y$.

By choosing a sampling pitch $N_{y}$ close to the grating pitch, $N_{y}$-step phase-shifting sampling moiré fringes can be digitally generated after performing down-sampling and intensity interpolation (linear or 2nd-order or 3rd-order) to the grating image (Fig. 2). The intensity of sampling moiré fringes can be expressed as

$$
\begin{aligned}
I_{m y}\left(k_{y}\right) & =A_{y} \cos \left[2 \pi\left(\frac{y}{P_{y}}-\frac{y}{N_{y}}+\frac{k_{y}}{N_{y}}\right)\right]+B_{y} \\
& \left.=A_{y} \cos \left[\varphi_{m y}+2 \pi \frac{k_{y}}{N_{y}}\right)\right]+B_{y} \quad\left(k_{y}=0,1, \ldots, N_{y}-1\right)
\end{aligned}
$$

where $k_{\mathrm{y}}$ is the starting point of the down sampling process, and $\varphi_{m y}$ signifies the phase of moiré fringes when $k_{y}=0$ generated from grating $Y$ in the $y$ direction.

The phase of moiré fringes at room temperature $T r$ when $k_{y}=0$ in the $y$ direction can be determined from the spatial phase-shifting technique using a discrete Fourier transform (DFT) algorithm, as shown in

$\varphi_{m y}=-\arctan \frac{\sum_{k_{y}=0}^{N_{y}-1} I_{m y}\left(k_{y}\right) \sin \left(2 \pi k_{y} / N_{y}\right)}{\sum_{k_{y}=0}^{N_{y}-1} I_{m y}\left(k_{y}\right) \cos \left(2 \pi k_{y} / N_{y}\right)}$

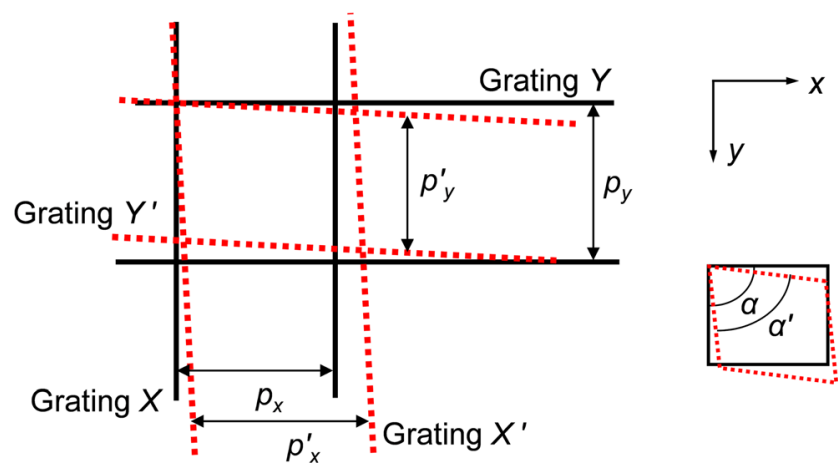

Fig. 1 Diagram of a 2D grating before (solid line) and after (dotted line) deformation 
(a) Grating

(b) Moiré fringes

(c) Moiré phase

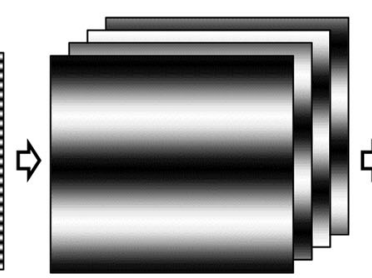

$\operatorname{Tr}$
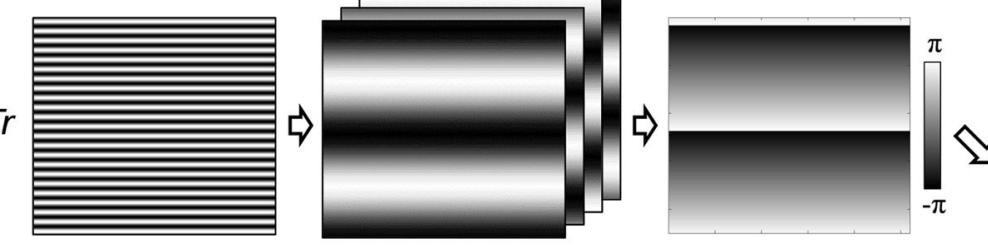

$T t$
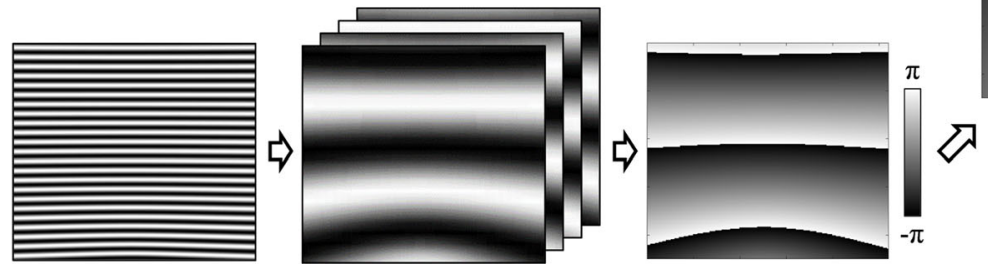

(d) Phase difference relative to $\mathrm{Tr}$ (e) Strain $y$

At $T r$ relative to $T t$

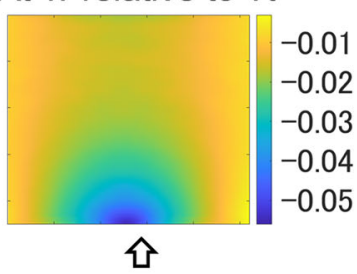

At $T t$ relative to $T r$
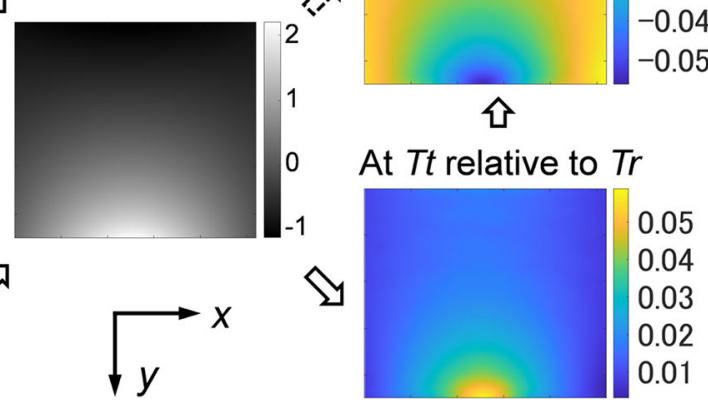

Fig. 2 Strain and residual measurement principle based on the sampling moiré technique

When the specimen temperature changes to arbitrary temperature Tt, grating $Y$ will change to grating $Y^{\prime}$ with the pitch of $P_{y}^{\prime}$ in the $y$ direction (Fig. 1). By substituting $P_{y}^{\prime}$ into Eqs. (1)-(3), the phase of moire fringes $\varphi_{m y}^{\prime}$ at $T t$ is also obtainable. The moiré phase difference in the $y$ direction at $T t$ relative to $\operatorname{Tr}$ can be calculated by

$$
\Delta \varphi_{m y}=\varphi_{m y}^{\prime}-\varphi_{m y}
$$

Similarly, sampling moiré fringes can also be generated from grating $X$, and the moiré phase difference in the $x$ direction at $T t$ relative to $T r$ can also be calculated using the above equations by replacing the direction symbol ' $y$ ' with ' $x$ '.

\section{Thermal Strain Measurement Relative to Room Temperature}

The two-dimensional (2D) thermal strain distributions can be measured from the moire phase differences in the $x$ and $y$ directions which are theoretically equal to the grating phase differences. In this study, the 2D strain distributions will be measured by the 2D moiré phase analysis method [21] which can be simplified when one grating is parallel to the analysis direction. Besides, to minimize the influence of defects on strain distributions, the local phase unwrapping algorithm [22] will be used to revise the partial differentials of moiré phase differences for strain measurement. From the revised partial differentials of moiré phase differences, the 2D thermal strains can be acquired by:

$$
\begin{aligned}
\varepsilon_{x} & =-\frac{1}{2 \pi} \frac{\partial \Delta \varphi_{m x}}{\partial x} P_{x} \\
\varepsilon_{y} & =-\frac{1}{2 \pi} \frac{\partial \Delta \varphi_{m y}}{\partial y} P_{y} \\
\gamma_{x y} & =-\frac{1}{2 \pi}\left(\frac{\partial \Delta \varphi_{m x}}{\partial y} P_{x}+\frac{\partial \Delta \varphi_{m y}}{\partial x} P_{y}\right)
\end{aligned}
$$

where $\varepsilon_{x}, \varepsilon_{y}, \gamma_{x y}$ respectively denote the strain in the $x$ direction, the strain in the $y$ direction and the shear strain at an arbitrary temperature $T t$ relative to room temperature $T r$.

\section{Residual Thermal Strain Measurement}

The measurement procedure for residual strain using an inverse approach is depicted in Fig. 3. Since the normal strain in the $x$ direction is obtainable from the pitch change, i.e., $\varepsilon_{x}=\left(p^{\prime}{ }_{x}-p_{x}\right) / p_{x}$, the thermal strains in the $x$ direction at $T t$ and $T f$ relative to $T r$ can be expressed from the relationship among the grating pitches

$$
\begin{aligned}
& \varepsilon_{x(T t)}=\frac{P_{x(T t)}-P_{x(T r)}}{p_{x(T r)}} \\
& \varepsilon_{x(T f)}=\frac{P_{x(T f)}-P_{x(T r)}}{P_{x(T r)}}
\end{aligned}
$$

where $T t$ means an arbitrary temperature, $T r$ is the grating formation temperature (usually the room temperature), and $T f$ expresses the specimen formation temperature.

Based on Eq. (6), as the residual normal strain at $T t$ relative to $T f$ can also be expressed from the pitch change, the residual strain in the $x$ direction at $T t$ is measurable from the thermal strains in the $x$ direction at $T t$ and $T f$ using the following equation

$\varepsilon_{x(T t) \_\mathrm{R}}=\frac{P_{x(T t)}-P_{x(T f)}}{P_{x(T f)}}=\frac{\varepsilon_{x(T t)}-\varepsilon_{x(T f)}}{1+\varepsilon_{x(T f)}}$

where $\varepsilon_{x(T t)}$ and $\varepsilon_{x(T f)}$ can be calculated by Eq. (5) using the moiré phase differences in the $x$ direction at $T t$ and $T f$ relative to $T r$, respectively. 


\section{Grating images}

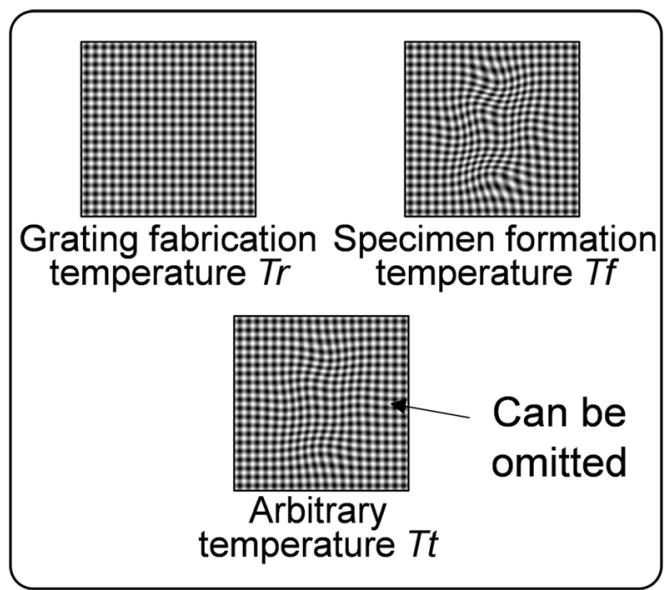

Phase difference

relative to $\mathrm{Tr}$
Residual strain

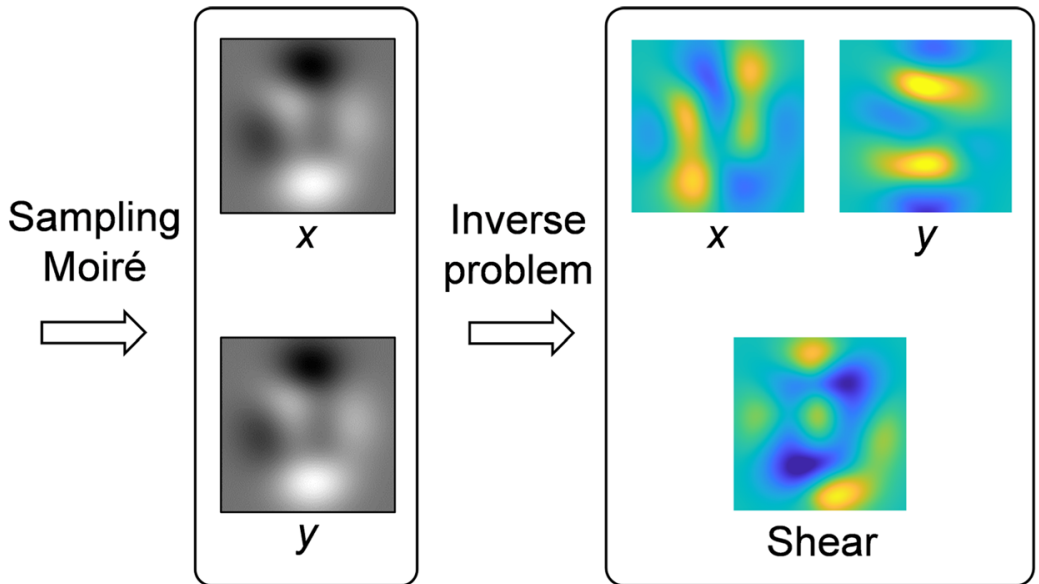

Fig. 3 Procedure for residual thermal strain measurement, where the grating fabrication temperature $\operatorname{Tr}$ usually refers to room temperature

Similarly, the residual strain in the $y$ direction at $T t$ is also obtainable from the thermal strains in the $y$ direction at $T t$ and $T f$ from the following equation

$\varepsilon_{y(T t) \_\mathrm{R}}=\frac{\varepsilon_{y(T t)}-\varepsilon_{y(T f)}}{1+\varepsilon_{y(T f)}}$

where $\varepsilon_{y(T t)}$ and $\varepsilon_{y(T f)}$ can be calculated by Eq. (5) using the moiré phase differences in the $y$ direction at $T t$ and $T f$ relative to $T r$, respectively.

Since the shear strain means the change of the intersection angle of the specimen grating, i.e., $\gamma_{x y}=-\left(\alpha^{\prime}-\alpha\right)$, where $\alpha^{\prime}$ stands for the angle after deformation, and $\gamma_{x y}$ is positive if the original right angle changes to an acute angle. The thermal shear strains at $T t$ and $T f$ relative to $T r$ can be represented by

$\gamma_{x y(T t)}=-\left(\alpha_{(T t)}-\alpha_{(T r)}\right)$

$\gamma_{x y(T f)}=-\left(\alpha_{(T f)}-\alpha_{(T r)}\right)$

Based on Eq. (9), as the residual shear strain at $T t$ relative to Tf can also be obtained from the angle change, the residual shear strain at $T t$ is measurable from the thermal shear strains at $T t$ and $T f$ using the following equation

$\gamma_{x y(T t) R}=-\left(\alpha_{(T t)}-\alpha_{(T f)}\right)=\gamma_{x y(T t)}-\gamma_{x y(T f)}$

where $\gamma_{x y(T t)}$ and $\gamma_{x y(T f)}$ can be calculated by Eq.(5) using the moiré phase differences in the $x$ and $y$ direction at $T t$ and $T f$ relative to $T r$, respectively.

Consequently, the residual normal strains in the $x$ and $y$ directions at an arbitrary temperature relative to the specimen formation temperature are measurable using Eqs. (7), (8) and (10), where the thermal strains at $T t$ and $T f$, i.e., $\varepsilon_{x(T t)}, \varepsilon_{y(T t)}$, $\gamma_{x y(T t)}, \varepsilon_{x(T f)}, \varepsilon_{y(T f)}$ and $\gamma_{x y(T f)}$ can be calculated from Eq. (5).

Note that Eqs. (7), (8) and (10) mean the residual strains relative to the specimen formation temperature ignoring the coefficient of thermal expansion (CTE). The residual strain relative to the free contraction state at any temperature can also be calculated from linear transformation of Eqs. (7), (8) and (10) by considering CTE. In defining the residual strain, the stress-free state was used as the reference state in [23], and the free contraction state refers to the stress-free state in this manuscript. In some cases, the residual strain distribution relative to the free contraction state is difficult to be plotted as the CTEs of different materials in a composite structure are different. For example, the CTEs of UF, die and the buffer layer are different, and the CTEs of resin and filler are also different inside the UF.

In the case that CTE is available, by considering the influence of CTE, Eqs. (7) and (8) can be revised for residual strain measurement in the $x$ and $y$ directions. To simplify the symbols of variables and directions, we simply use $P$ to express the grid pitch at the specimen formation temperature $T f$ in the $x$ direction '(Fig. 4). When the temperature becomes an arbitrary temperature $T t$, the grid pitch changes to $P+\Delta P_{\mathrm{R}}=(1+$ $\left.\varepsilon_{x(T t) \_\mathrm{R}}\right) P$ in the actual state with residual stress due to constrains, where $\varepsilon_{x(T t) \_ \text {R }}$ can be obtained from Eqs. (7) and (8) in different directions.

If the material can be freely shrunk without any restriction when temperature decreases to $T t$ from the formation temperature, theoretically there is no residual stress even though the grid pitch changes. In the case of free shrinkage, the grid pitch will become $P+\Delta P_{\alpha}=\left(1+\int_{T f}^{T t} \alpha d T\right) P$, where $\alpha$ stands for the CTE. Then the revised residual strain relative to the state after free contraction at $T t$ in the $x$ direction can be calculated from the change of the grid pitch as follow:

$$
\begin{aligned}
\varepsilon_{x(T t) \_ \text {R_rev }} & =\frac{\left(P+\Delta P_{\mathrm{R}}\right)-\left(P+\Delta P_{\alpha}\right)}{\left(P+\Delta P_{\alpha}\right)}=\frac{\left(1+\varepsilon_{x(T t) \_\mathrm{R}}\right) P-\left(1+\int_{T f}^{T t} \alpha d T\right) P}{\left(1+\int_{T f}^{T t} \alpha d T\right) P} \\
& =\frac{\varepsilon_{x(T t) \_\mathrm{R}}-\int_{T f}^{T t} \alpha d T}{1+\int_{T f}^{T t} \alpha d T}
\end{aligned}
$$


Similarly, the revised residual strain relative to the state after free contraction at $T t$ in the $y$ direction can be measured using the same manner:

$\varepsilon_{y(T t) \_\mathrm{R} \_\mathrm{rev}}=\frac{\varepsilon_{y(T t) \_\mathrm{R}}-\int_{T f}^{T t} \alpha d T}{1+\int_{T f}^{T t} \alpha d T}$

In Eqs. (11)-(12), $\varepsilon_{x(T t) \mathrm{R}}$ and $\varepsilon_{y(T t) \mathrm{R}}$ can be calculated using Eqs. (7)-(8). The residual shear strain can also be measured using Eq. (10) as the grid intersection angle stays the same before and after free contraction.

\section{Principal Residual Strain Measurement}

After the residual strains are measured, the residual principal strains can be determined from the analysis of strain status. For plane-stress problems, the equations for calculating the residual principal strains are as follows

$$
\begin{aligned}
& \varepsilon_{\max }=\frac{\varepsilon_{x}+\varepsilon_{y}}{2}+\sqrt{\left(\frac{\varepsilon_{x}-\varepsilon_{y}}{2}\right)^{2}+\frac{\gamma_{x y}^{2}}{4}} \\
& \varepsilon_{\min }=\frac{\varepsilon_{x}+\varepsilon_{y}}{2}-\sqrt{\left(\frac{\varepsilon_{x}-\varepsilon_{y}}{2}\right)^{2}+\frac{\gamma_{x y}^{2}}{4}}
\end{aligned}
$$

When the thermal strains $\varepsilon_{x}, \varepsilon_{y}$ and $\gamma_{x y}$ relative to the room temperature in Eq. (5) are substituted into Eq. (13), the principal thermal strains are obtainable. When the residual strains $\varepsilon_{x(T t) \_ \text {R rev, }} \varepsilon_{y(T t) \_ \text {R rev }}$ and $\gamma_{x y(T t) \_\mathrm{R}}$ in Eqs. (11), (12) and (10) are substituted into Eq. (13), the principal residual strains relative to the specimen formation temperature are measurable.

Besides, the principal residual stresses can be further measured using Hooke's law for plane-stress problems when Young's modulus and the Poisson's ratio are known.

\section{Simulation Verification and Method Discussion}

\section{Simulation Verification of Residual Strain Measurement}

This section is used to verify the measurement accuracy of the image processing part of the proposed technique for measuring the residual normal and shear strains from the numerical simulation. Since the residual strains before and after considering CTE have a linear relationship, the residual strains ignoring CTE will be calculated in this simulation section. The used 2D grids and load conditions are illustrated in Fig. 5(a). The grating pitches in both the $x$ and $y$ directions are 10 pixels, and the total image size is $370 \times 570$ pixels. A random noise with the amplitude of $\sigma=2 \%$ of the grating amplitude was added to the grating.

The grating at state $T_{1}$ was deformed to the grating at state $T_{2}$ by exerting normal and shear strains. The grating at state $T_{2}$ could be transformed to the grating at state $T_{1}$ by applying residual normal strains in the $x$ and $y$ directions and residual shear strain. The theoretical residual strains were known from the strain Mohr's circle and the residual strain Mohr's circle, a little bit different with the opposite values of the theoretical strains. A series of theoretical residual strains were applied to the grating at state $T_{2}$.

The residual strains of this grating were measured using the proposed technique, and the relationship between the measured average residual strains and the theoretical residual strains were plotted in Fig. 5(b). It can be seen that the measured residual strains agree well with the theoretical residual strains. The absolute errors, the relative errors and the standard deviations of the measured residual strains along with the theoretical residual strains were listed in Fig. 6. With the
Fig. 4 Schematic diagram of residual strain transformation, where $P$ means the grid pitch in the $x$ direction, $T f$ is the specimen formation temperature and $T t$ is an arbitrary temperature

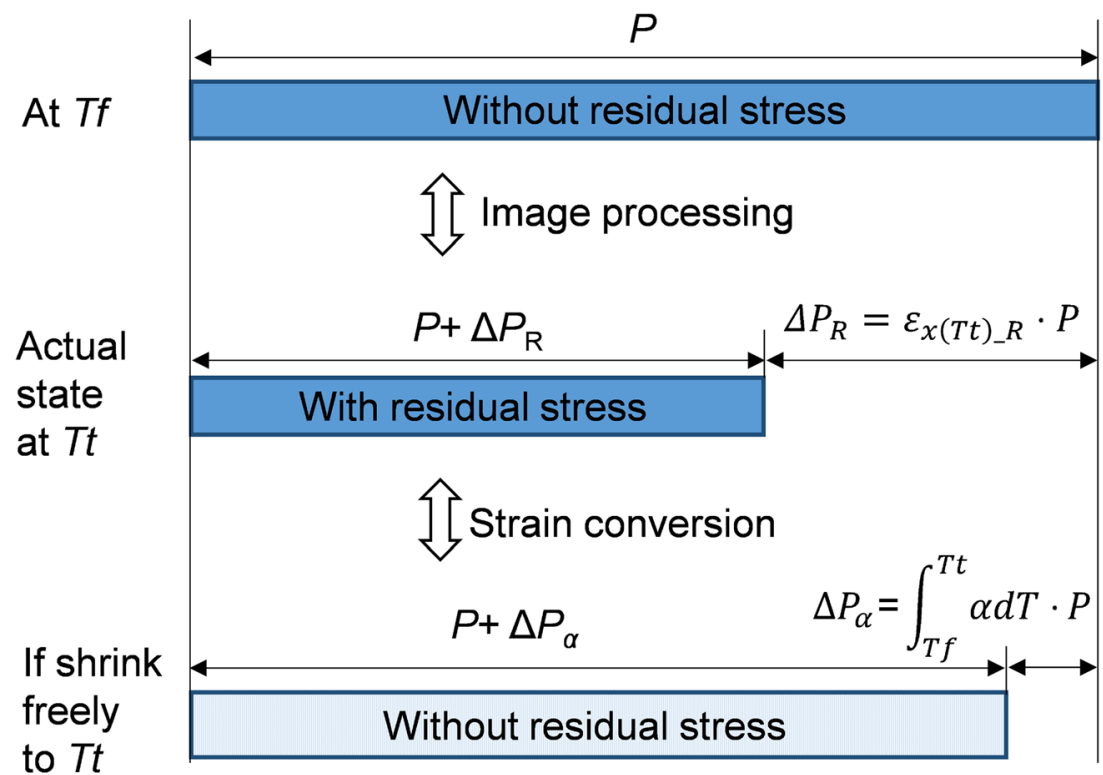


Fig. 5 Simulation conditions: (a) simulated gratings at different temperatures and (b) comparison between the measured and theoretical residual strains

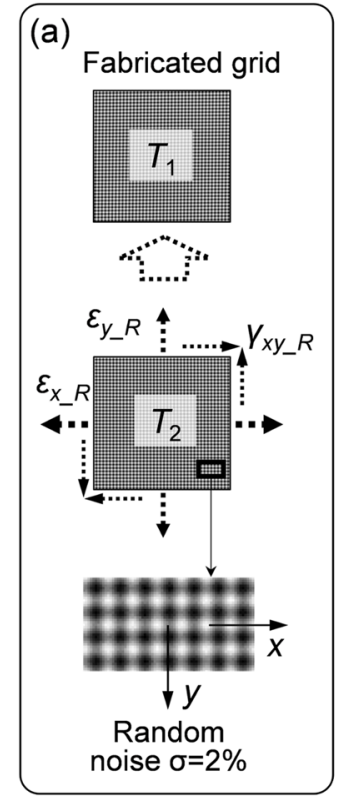

(b) Residual strain

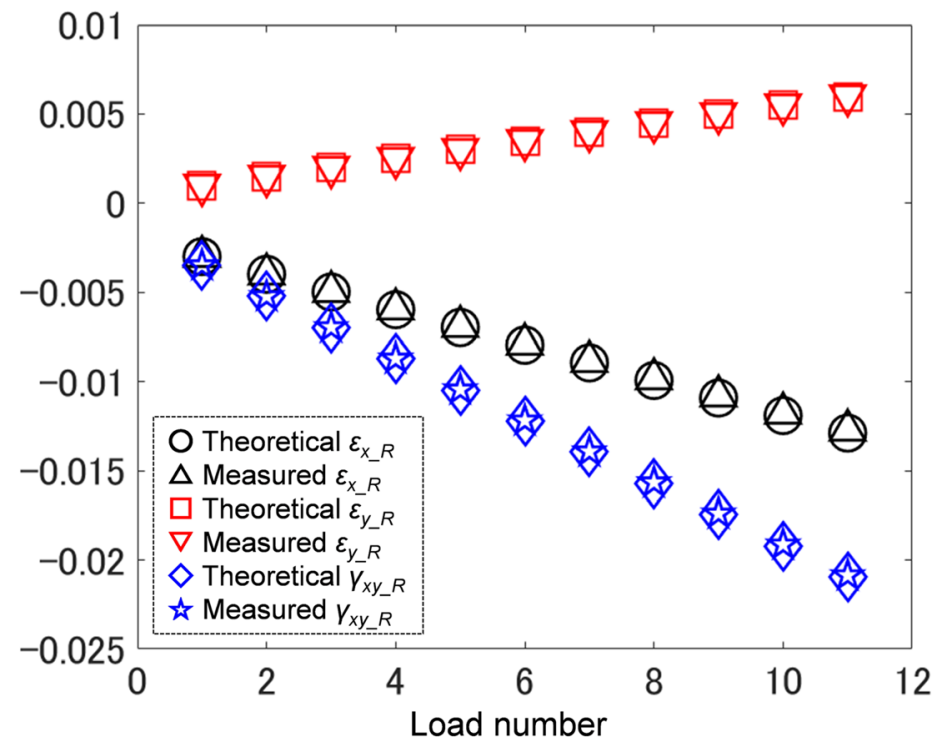

increase of the absolute value of the theoretical residual strain, the absolute value of the absolute strain errors and the relative strain errors also increase. All the absolute errors were within -0.00017 to 0 , and all the relative errors range from $-1.5 \%$ to $0.7 \%$, indicating the high accuracy of the proposed technique for residual strain measurement. All the standard deviations were less than 0.0012 for the residual strain measurement, showing the small discreteness and the good stability of the proposed technique for residual strain measurement even when the used grating has a random noise.
Fig. 6 Simulation results of the (a) absolute error, (b) relative error and (c) standard deviation of measured residual strain (a)

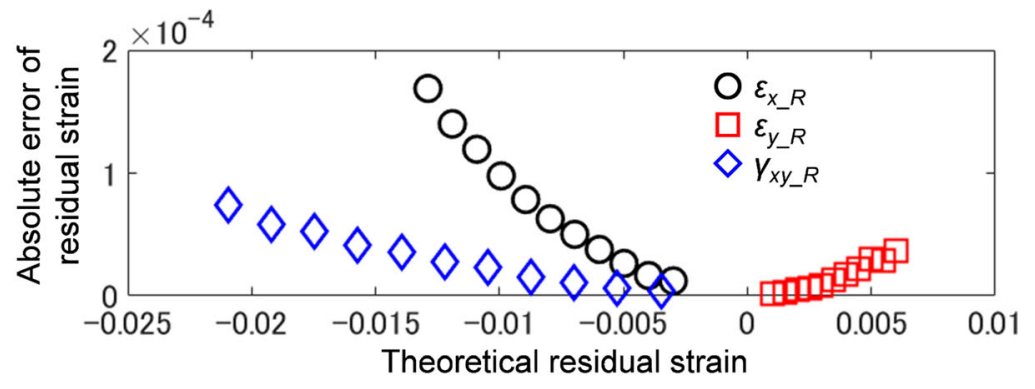

(b)

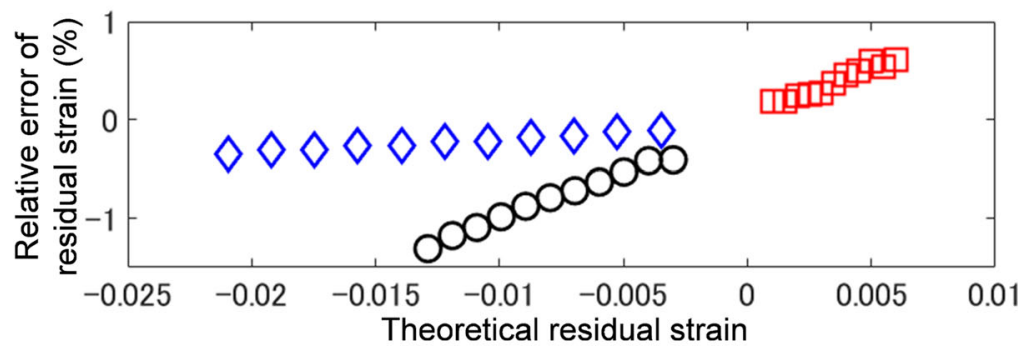

(c)

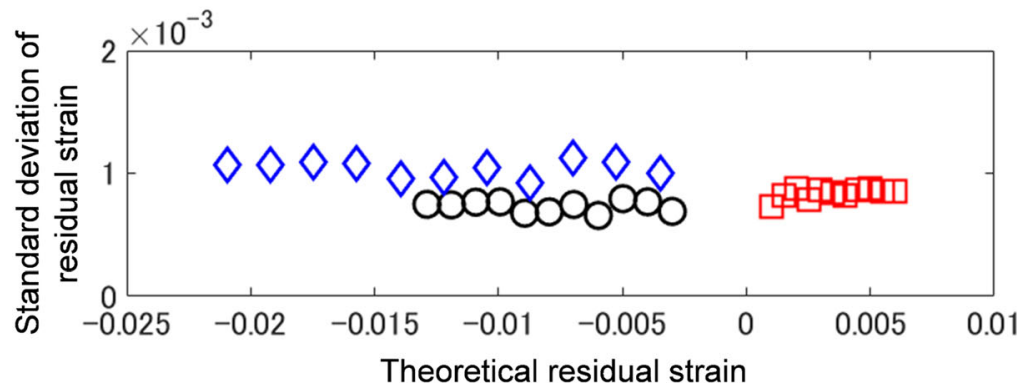


Fig. 7 FCPKG specimen preparation (a) designed clamping jig and specimen geometry, (b) grid fabrication process using UV nanoimprint lithography

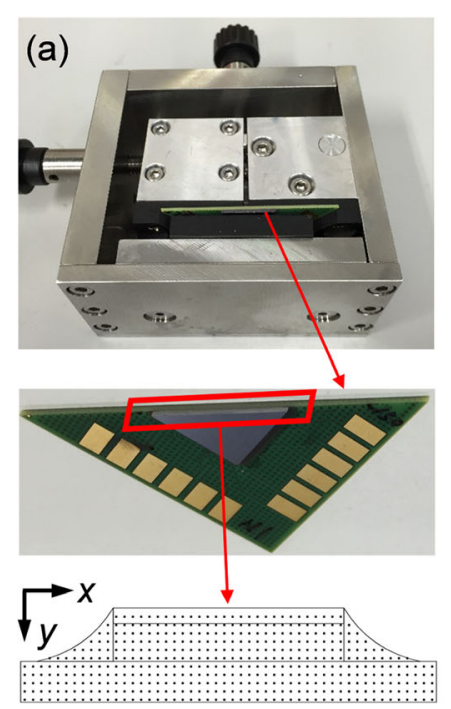

(b)
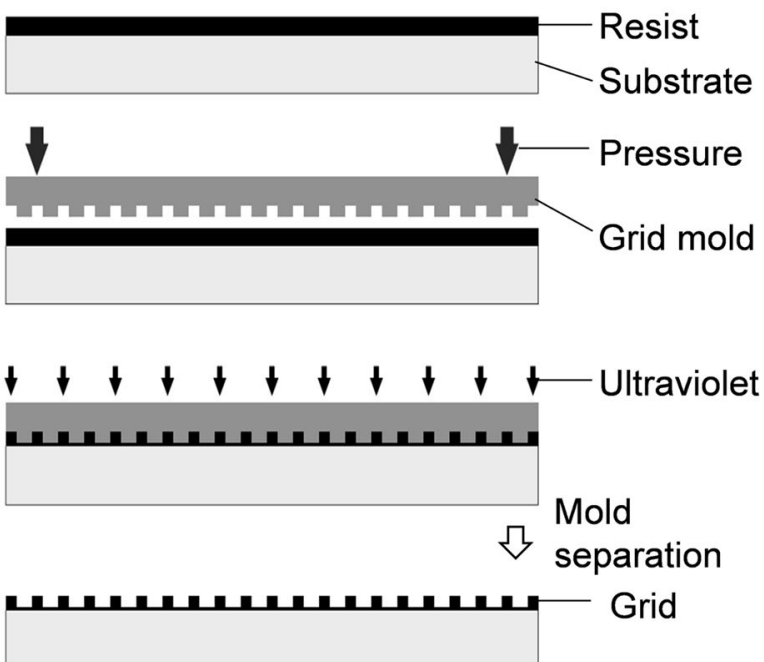

\section{Discussion of this Method}

Compared with conventional point strain measurement sensor and other full-field imaging techniques, the advantages of the proposed moiré technique mainly include:
1) Full-field residual strain measurements: The precise distributions of 2D residual strains including normal, shear and principal strains are simultaneously measurable;

2) Fast and accurate: High accuracy, automatic batch processing and fast image storage for displacement, strain, residual strain and residual stress measurements;
Fig. 8 Experimental setup for thermal test of FCPKG: (a) designed thermal chamber under a laser microscope, and (b) observation section of the thermal chamber. The 5-axis stage can control the back-forth and leftright tilt motions, and the in-plane rotation as well as translational motions in $x$ and $y$ directions. The movement in the $z$ direction can also be controlled by the microscope
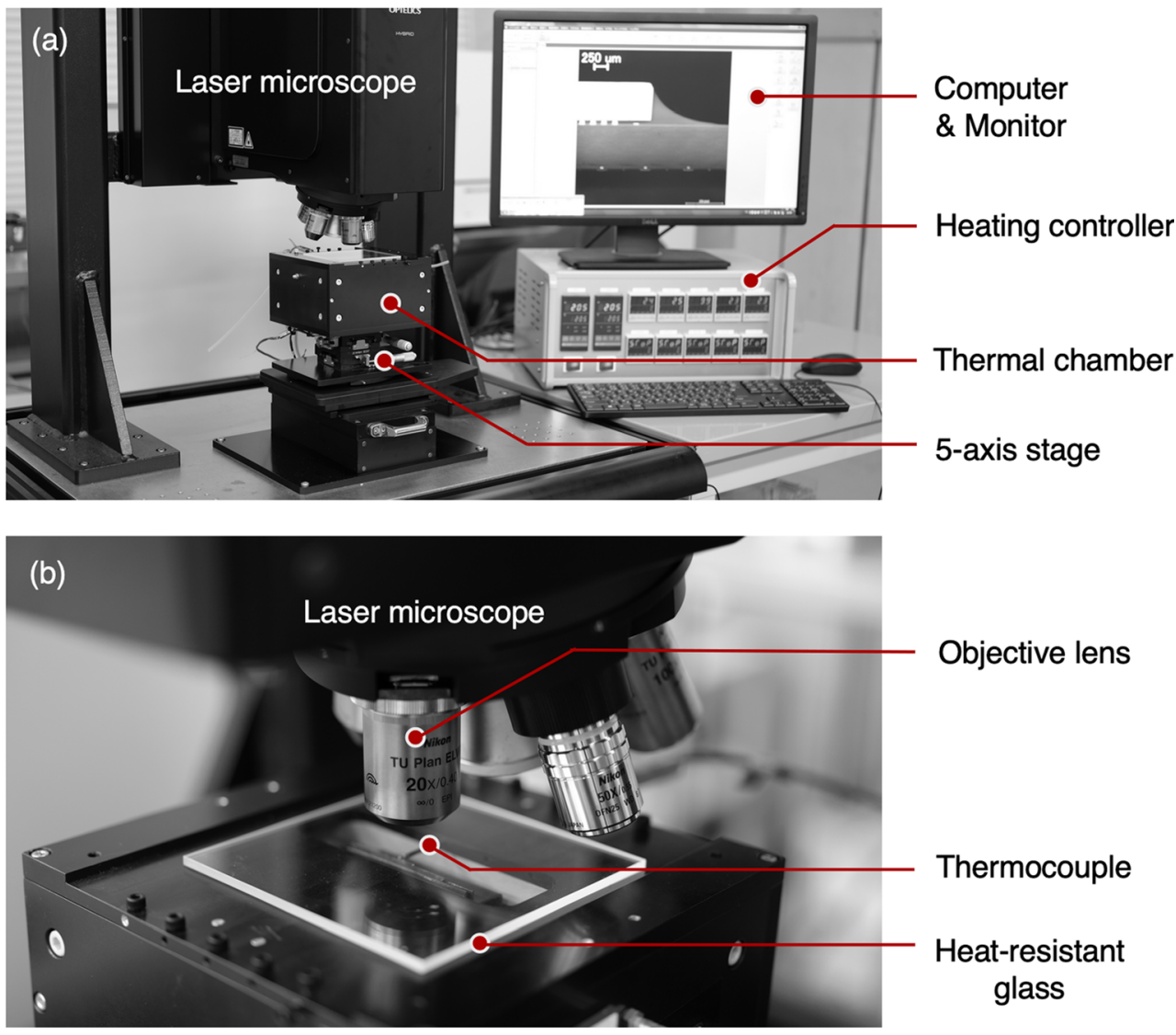
3) Dynamic deformation measurement: The proposed moiré technique is suitable for dynamic deformation measurement and able to display results in real time;

4) Wide strain measurement range: Both large deformation and small deformation can be accurately measured even if the periodic pattern is inclined at a large angle.

The proposed residual deformation measurement technique can be used in industrial fields such as aerospace, automobiles, electronic packages, biomedicine, military, material manufacturing, etc. It is mainly useful for failure analysis, residual stress measurement, strengthening and toughening of materials ranging from nanoscale to meter-scale, optimal design of interfaces, production quality control, structural health monitoring, etc.

Besides, the inverse approach can also be combined with other grid-based methods for residual strain measurement, including geometric phase analysis (GPA) using Fourier transform (FT) or windowed Fourier transform (WFT). Compared with GPA using FT, the sampling moiré method uses local phase information, and is more suitable for objects with complex boundaries. Compared with GPA using WFT, the sampling moiré method has almost the same measurement accuracy but has much higher calculation speed [24].

\section{Experiments to Flip Chip Packages}

\section{Specimen Preparation and Grid Fabrication}

Two FCPKGs containing two different kinds of UFs formed at $150{ }^{\circ} \mathrm{Cwere}$ used as the objects of study, where the UF having low glass transition temperature $(T g)$ was called as UF-A, and the other one having high $T g$ was referred to as UF-B. These two square FCPKGs with a size of $25 \times 25 \mathrm{~mm}^{2}$ were cut along their diagonal lines, and half of both were used as two triangular specimens containing UF-A and UF-B, respectively. The cross sections of the specimens were polished with sandpapers and polishing solution successively.

For grid fabrication on the polished cross-sections, a clamping jig used in an ultraviolet (UV) nanoimprint device (EUN-4200) was designed to fix the specimens, as shown in Fig. 7(a). The height of the clamping jig was $38 \mathrm{~mm}$, the total length including the back-forth movement adjustment knob
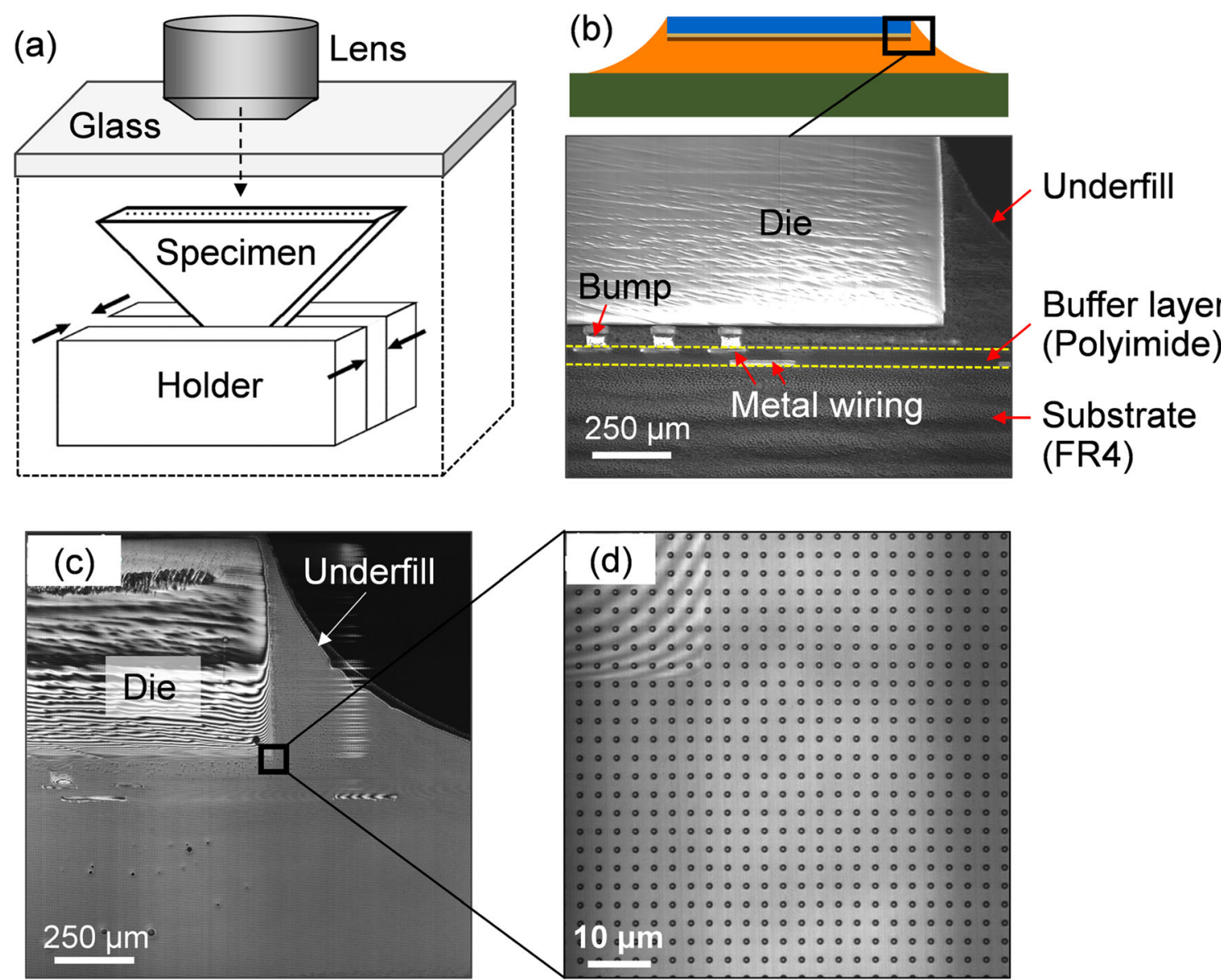

Fig. 9 Observation of the fabricated grid on the cross section of FCPKG with UF-A: (a) diagram of specimen clamping, (b) cross section image before grid fabrication, (c) grid image in a large field of view around a die corner, and (d) enlarged view of the grid image 
was $107 \mathrm{~mm}$ and the total width including the left-right movement adjustment knob was $98 \mathrm{~mm}$. Soft rubber materials were used around the specimens in the clamping jig for specimen protection and convenient adjustment of self-balancing to fit the flat nanoimprint plate.

On the cross section of the specimens, a kind of UV nanoimprint resist PAK01 was dropped and spin-coated. Since the thermally stable temperature for PAK01 is approximately $270{ }^{\circ} \mathrm{C}$ [25], it can be used for deformation measurement of specimens lower than $150{ }^{\circ} \mathrm{C}$. Then, a grid with a pitch of $3 \mu \mathrm{m}$ was fabricated on the cross section using UV nanoimprint lithography at $25{ }^{\circ} \mathrm{C}$ (room temperature). The grid fabrication process of UV nanoimprint lithography was drawn in Fig. 7(b). The pressure was $0.2 \mathrm{MPa}$, the used UV wavelength was $375 \mathrm{~nm}$, and the UV illumination time was 30s. The grid mold was from SCIVAX Corporation. Next, the grid on either FCPKG specimen was coated with a layer of platinum with a thickness of about $3 \mathrm{~nm}$ to increase the contrast of the grid image.

Compared with commercially available grid plate for moiré interferometry, the cost of the UV nanoimprint mold is not much different. However, the UV nanoimprint polymer mold is easy to cut and can be used for several times, and the grid can be quickly fabricated by UV nanoimprint lithography [26].

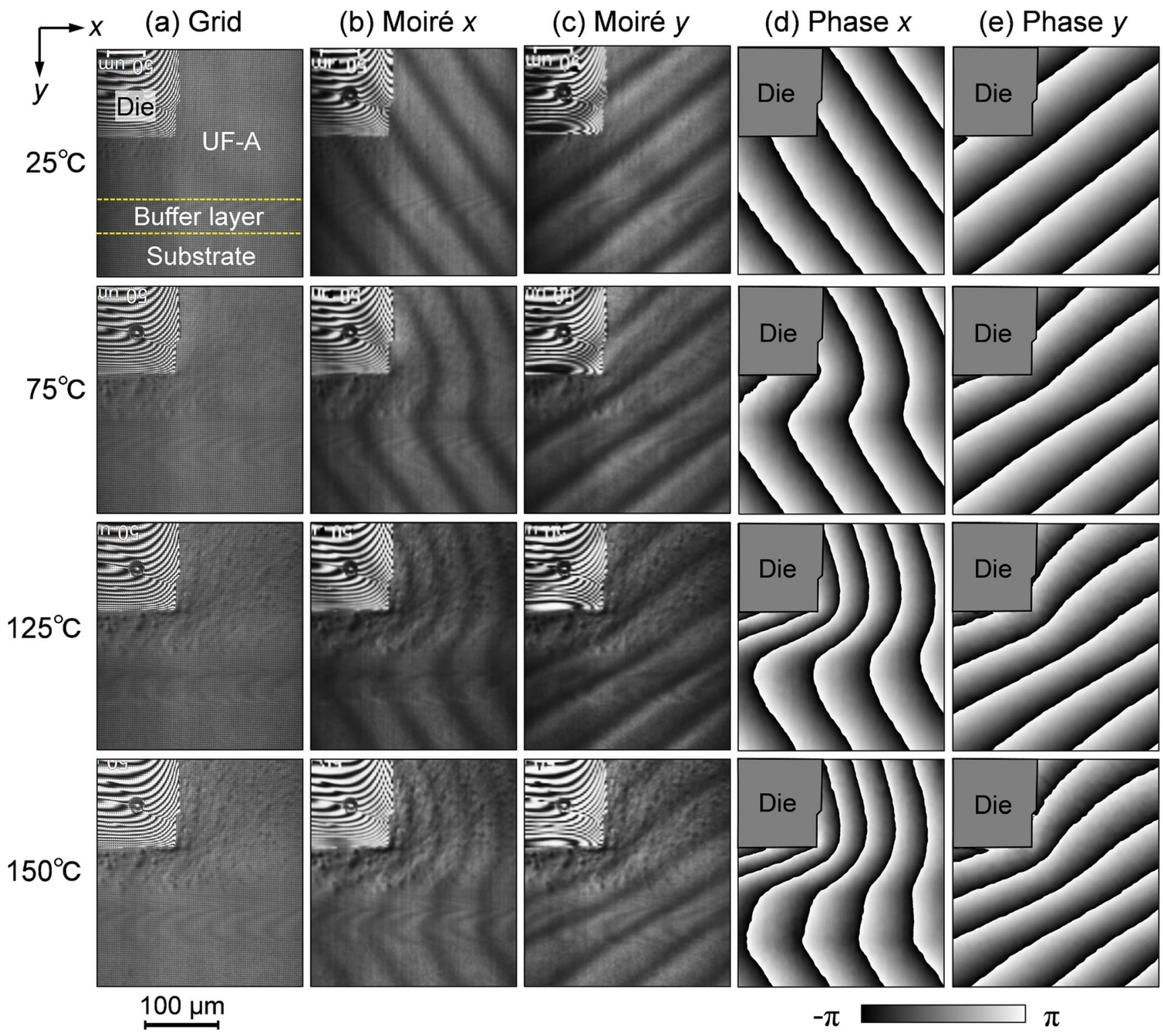

Fig. 10 Residual thermal strain measurement at different temperatures on FCPKG with UF-A: (a) grid images, moiré fringe patterns in (b) $x$ direction and (c) $y$ direction, and moiré phases in (d) $x$ direction and (e) $y$ direction 


\section{Heating Experiment under a Laser Microscope Observation}

To perform the heating experiment to the FCPKG specimens, a thermal chamber was designed under a laser scanning microscope (Lasertec, Optelics Hybrid), as illustrated in Fig. 8(a). The rectangular corner of either specimen was fixed, and the cross-section with the grid was observed by the laser microscope (Fig. 9(a)). A heat-resistance glass (Fig. 8(b)) with a thickness of $3 \mathrm{~mm}$ was placed at the top side of an observation window of the thermal chamber. The temperature of either specimen was measured with a thermal couple which touches the center of the die part. The other thermal couples do not touch the specimen to insure the free deformation of the specimen. The specimen temperature was controlled and monitored by a heating controller.

The thermal chamber was placed on a 5-axis stage, where the back-forth and left-right tilt angles are controllable by a tilt adjustment stage installed at the bottom side of the thermal chamber, and the in-plane rotation and translational motions in the $x$ and $y$ directions are adjustable by the affiliated sample stage of the laser microscope. Besides, the movement in the $z$ direction can also be implemented by the focus adjustment unit of the laser microscope. Thus, the heating system made up by the thermal chamber and the laser microscope can carry out 6-axis or 6-dimensional adjustment including 3dimensional translation and 3-dimensional rotation.

During the heating experiment, the temperature of either specimen was increased from $25{ }^{\circ} \mathrm{C}$ to $150{ }^{\circ} \mathrm{C}$, i.e., from the room temperature to the specimen formation temperature. The grid images on the cross section were recorded at $25{ }^{\circ} \mathrm{C}$, $75{ }^{\circ} \mathrm{C}, 125^{\circ} \mathrm{C}$ and $150{ }^{\circ} \mathrm{C}$ by the laser microscope. As the aim is to evaluate the residual strain of UF, we mainly focus on the area of UF around one corner of the die (Figs. 9(b) and $9(\mathrm{c}))$.

Note that some chemical changes in the FCPKG will occur and some gas will be released during the heating experiment. When the gas meets the glass, some misty liquid will be generated on the inner surface of the glass. To avoid the misty liquid area, during the image observation under the microscope, the glass whose plane view size is much greater than the observation window of the thermal chamber should be slightly moved. Unnoticed misty liquid area will affect the quality of the grid image. Fortunately, the moiré method has strong noise-resistance ability and the strain measurement is almost unaffected. (a) Strain $x$

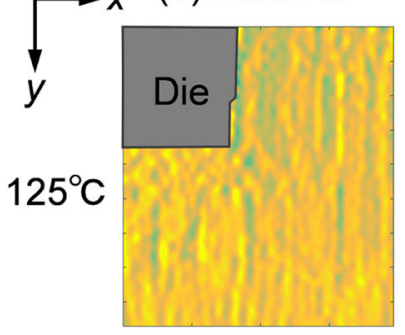

(b) Strain $y$
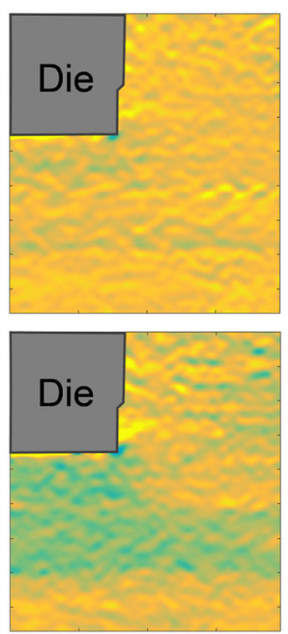

$75^{\circ} \mathrm{C}$
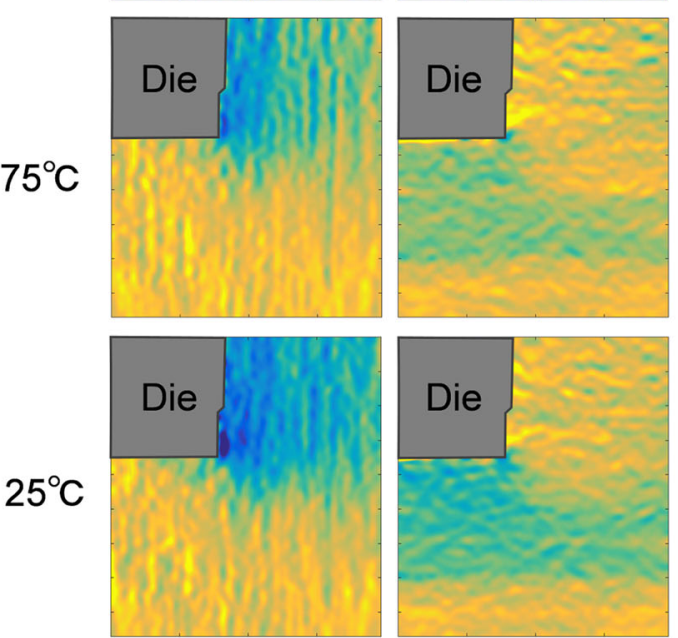

$100 \mu \mathrm{m}$

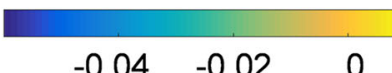

(c) Shear
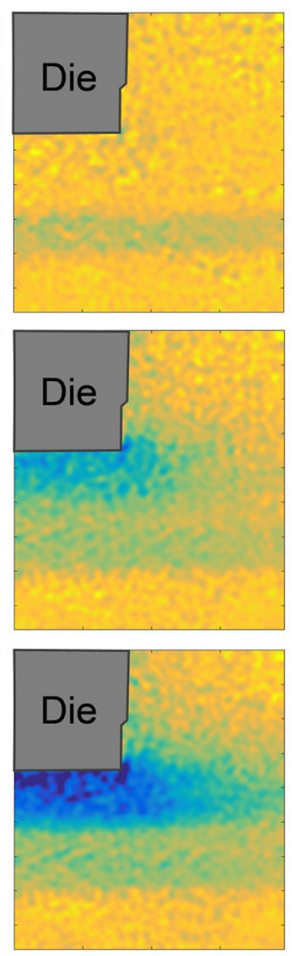

$-0.12-0.06 \quad 0$ (d) Maximum principal (e) Minimum principal
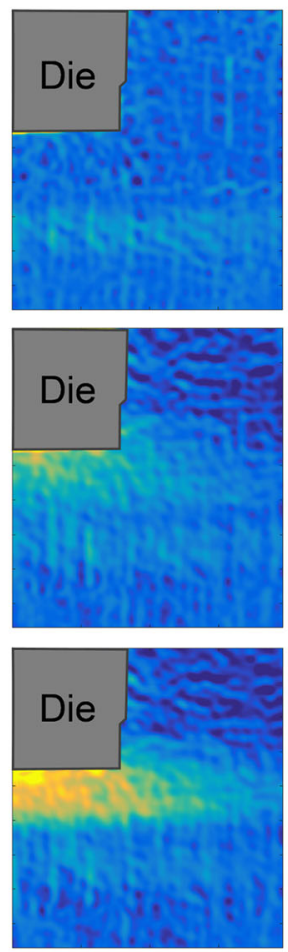

$0 \quad 0.02 \quad 0.04$
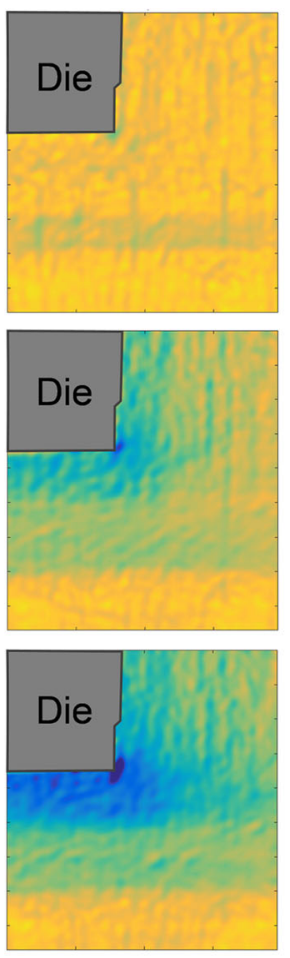

$-0.08 \quad-0.04 \quad 0$

Fig. 11 Residual thermal strain distributions of FCPKG with UF-A relative to $150^{\circ} \mathrm{C}$ : normal strains in (a) $x$ direction and (b) $y$ direction, (c) shear strain, and (d) maximum and (e) minimum principal strains 


\section{Residual Strain Distributions of Underfills}

For reference, the thermal displacement distributions of two types of underfills at $150{ }^{\circ} \mathrm{C}$ relative to $25^{\circ} \mathrm{C}$ are depicted in Appendix 1. Compared with the nano-pattern recognition and correlation technique [27], the proposed technique can measure not only the thermal deformation, but also the residual thermal deformation of materials. The residual strain distributions of both underfills relative to $150^{\circ} \mathrm{C}$ are measured using the proposed technique.

\section{Residual Strain Measurement of Underfill A}

This section shows the 2D residual thermal strain measurement results of the low- $\mathrm{Tg}(345 \mathrm{~K})$ underfill (UF-A) in an FCPKG using the proposed technique. Figures 9(b)-9(d) presents the laser microscope images of the FCPKG surface before and after grid fabrication and the enlarged images of the fabricated 3- $\mu \mathrm{m}$-pitch grid. The laser wavelength was $405 \mathrm{~nm}$ in the used laser microscope.

During the heating test, an area of $286 \times 316 \mu \mathrm{m}^{2}$ around one die corner (as shown in Fig. 10) on UF-A of this FCPKG was chosen as the region of interest. The grid images in this region of interest at $25^{\circ} \mathrm{C}, 75^{\circ} \mathrm{C}, 125^{\circ} \mathrm{C}$ and $150{ }^{\circ} \mathrm{C}$ were recorded, and the corresponding residual thermal strain distributions were measured using the proposed technique. The recorded grating images and the 8-pixel down-sampling moiré fringes in the $x$ and $y$ directions were illustrated in Figs. 10(a)-10(c) at those temperatures. The corresponding moiré phases of these moiré fringes in the $x$ and $y$ directions were then calculated, as shown in Figs. 10(d) and 10(e). The basic deformation features were visible in the moiré fringes and phase images.

From the phase differences relative to the phase at $25^{\circ} \mathrm{C}$, the distributions of $x$-direction, $y$-direction and shear strains of UF-A at $75{ }^{\circ} \mathrm{C}, 125{ }^{\circ} \mathrm{C}$ and $150{ }^{\circ} \mathrm{C}$ are obtainable. As the theoretical strain of this $\mathrm{FCPKG}$ is zero at $150^{\circ} \mathrm{C}$, the residual

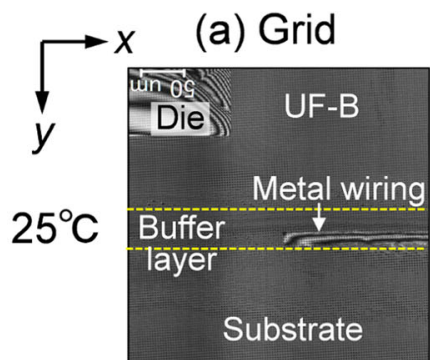

$75^{\circ} \mathrm{C}$

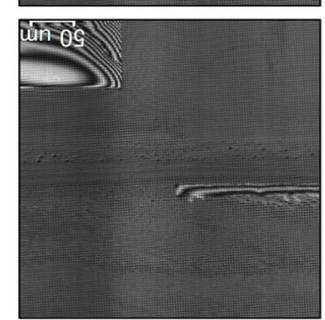

$125^{\circ} \mathrm{C}$
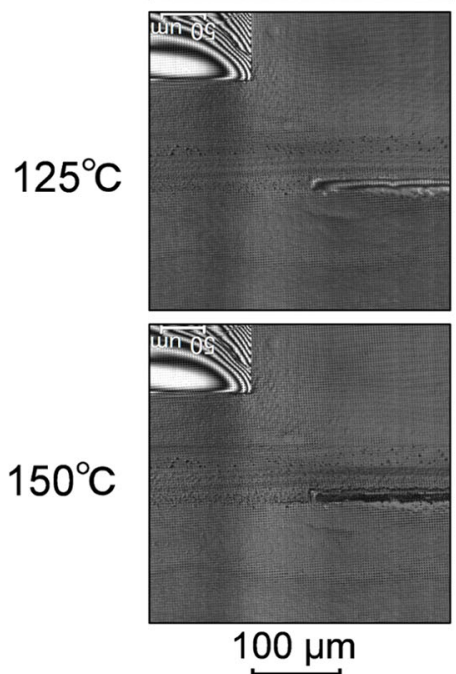

$100 \mu \mathrm{m}$ (b) Moiré $x$
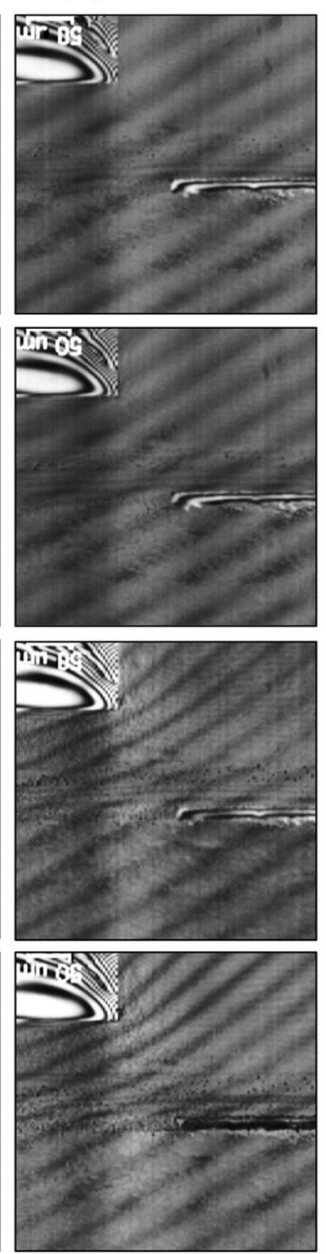

(c) Moiré $y$
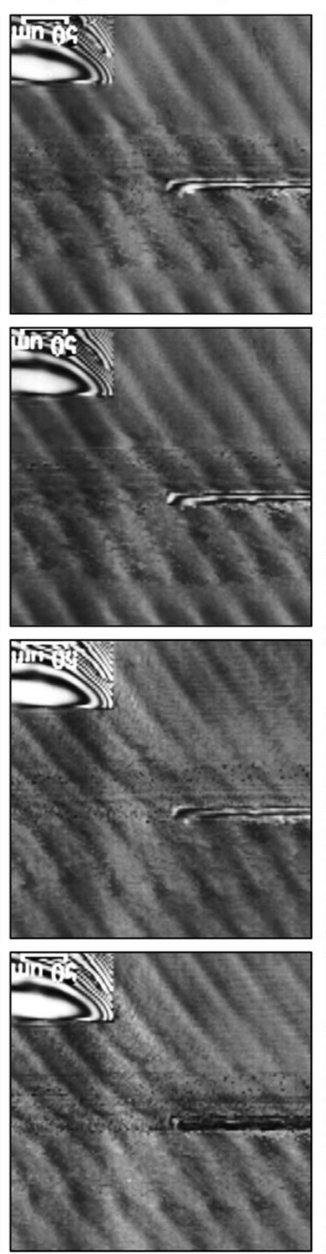

(d) Phase $x$
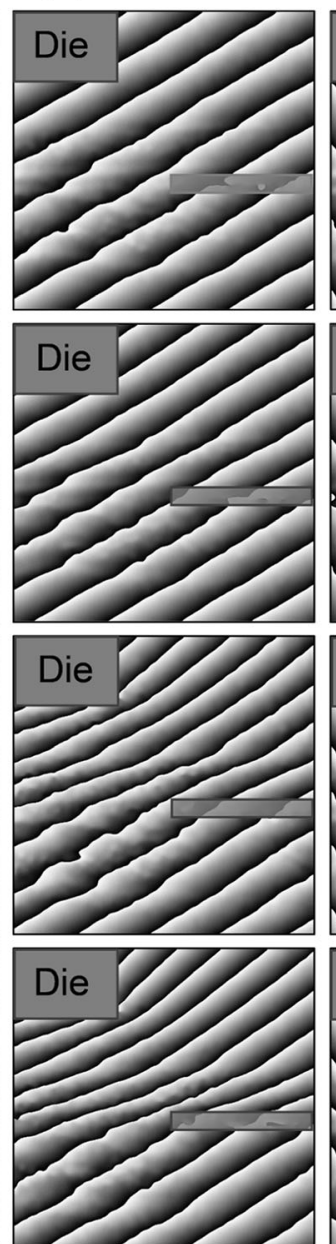

$-\pi$ (e) Phase $y$
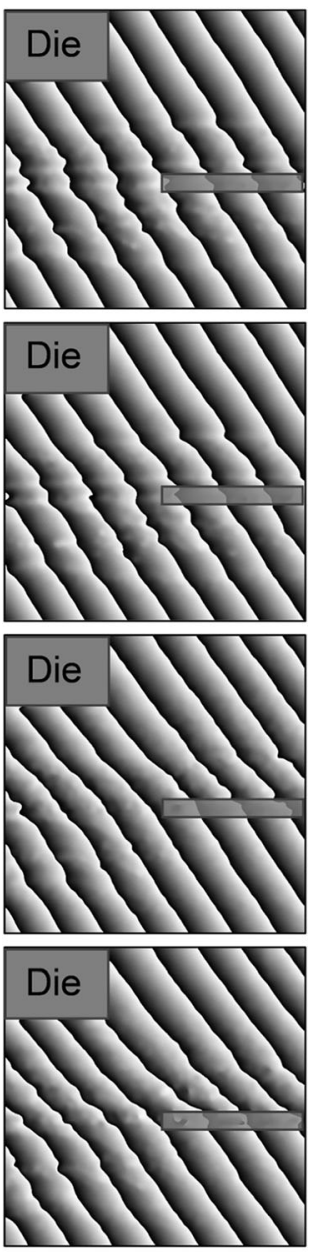

$\pi$

Fig. 12 Residual thermal strain measurement at different temperatures on FCPKG with UF-B: (a) grid images, moiré fringe patterns in (b) $x$ direction and (c) $y$ direction, and moiré phases in (d) $x$ direction and (e) $y$ direction 
strains of UF-A at other temperature relative to $150{ }^{\circ} \mathrm{C}$ can be measured using Eqs. (7), (8) and (10). The measured residual normal strain distributions in the $x$ and $y$ directions and residual shear strain distributions at $125^{\circ} \mathrm{C}, 75^{\circ} \mathrm{C}$ and $25^{\circ} \mathrm{C}$ are shown in Figs. 11(a)-11(c). The absolute value of the residual strain in the $x$ direction is highest at the right side of the die, especially at the corner area around the die. The absolute values of the residual strain in the $y$ direction and the residual shear strain are maximum at the bottom area below the die and the corner area around the die.

Using Eq. (13), the maximum and minimum residual principal strains (Figs. 11(d) and 11(e)) were also determined at $125^{\circ} \mathrm{C}, 75^{\circ} \mathrm{C}$ and $25^{\circ} \mathrm{C}$, respectively. The maximum principal residual strain is highest at the bottom area below the die, and the absolute value of the minimum principal strain is highest at the corner around the die.

\section{Residual Strain Measurement of Underfill B}

Using the similar measurement procedure, the $2 \mathrm{D}$ residual thermal strain distributions of the high- $T g(380 \mathrm{~K})$ underfill (UF-B) in another FCPKG were also measured. A 3- $\mu$ m-pitch grid was fabricated on the cross section of UF-B, using the same nanoimprint method as UF-A. The grid images at $25^{\circ} \mathrm{C}$, $75^{\circ} \mathrm{C}, 125^{\circ} \mathrm{C}$ and $150{ }^{\circ} \mathrm{C}$ in the region of interest, an area of
$354 \times 351 \mu \mathrm{m}^{2}$ around one die corner, are shown in Fig. 12(a). The corresponding moiré fringe patterns and the moiré phases at different temperatures in the $x$ and $y$ directions are also presented in Figs. 12(b)-(e), where the sampling pitch is 8 pixels.

Since the specimen formation temperature was also $150{ }^{\circ} \mathrm{C}$, the residual strain distributions including normal and shear strains were measured at $125^{\circ} \mathrm{C}, 75^{\circ} \mathrm{C}$ and $25^{\circ} \mathrm{C}$, as seen in Figs. 13(a)-13(c). The absolute value of the residual strain in the $x$ direction is higher at the right side of the die and greatest near the die corner. The residual strain in the $y$ direction and the residual shear strain mainly concentrate at the buffer layer, a little bit far from the die.

The calculated residual principal strains at $125^{\circ} \mathrm{C}, 75^{\circ} \mathrm{C}$ and $25{ }^{\circ} \mathrm{C}$ are depicted in Figs. 13(d) and 13(e). Both the maximum and minimum residual principal strains tend to concentrate at the buffer layer besides the corner.

\section{Comparation with FEM Simulation and Discussion}

The authors also performed a simulation study on the residual strains of these two kinds of UFs using the finite element method (FEM). Figure 14 shows the simulation model of the

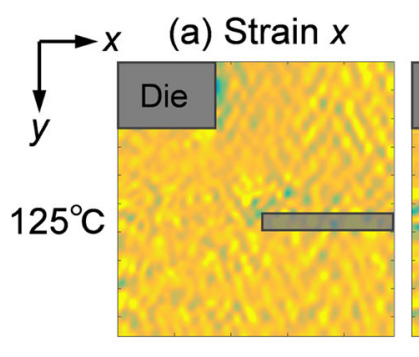

(b) Strain $y$
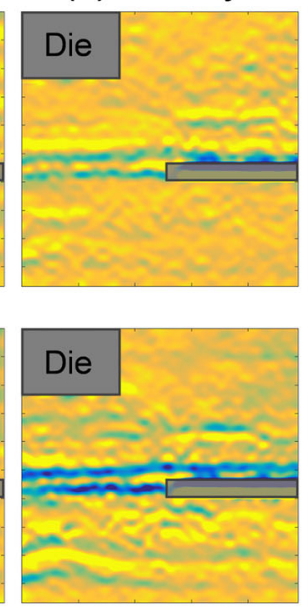

$75^{\circ} \mathrm{C}$
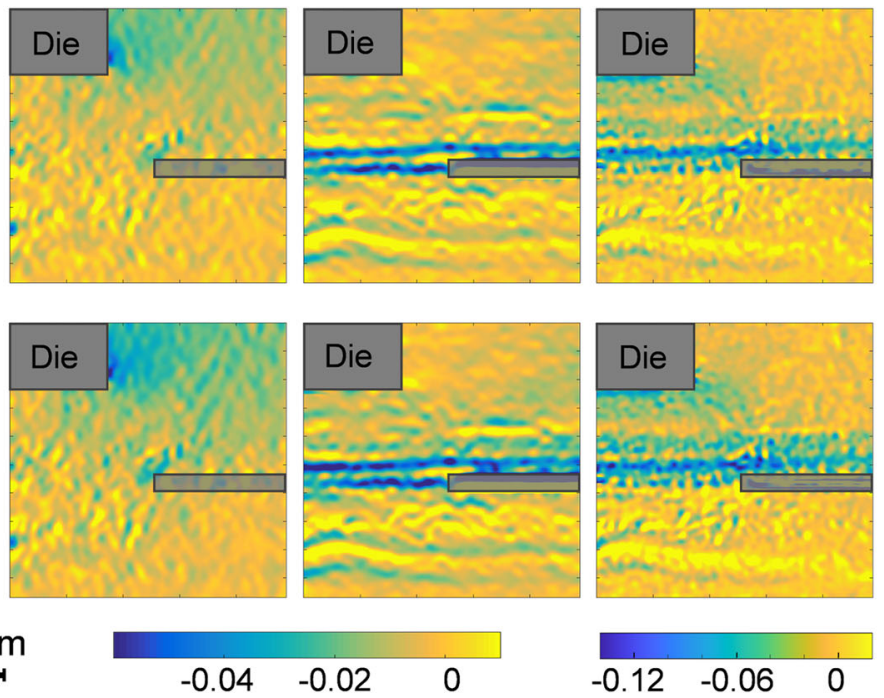

(d) Maximum principal
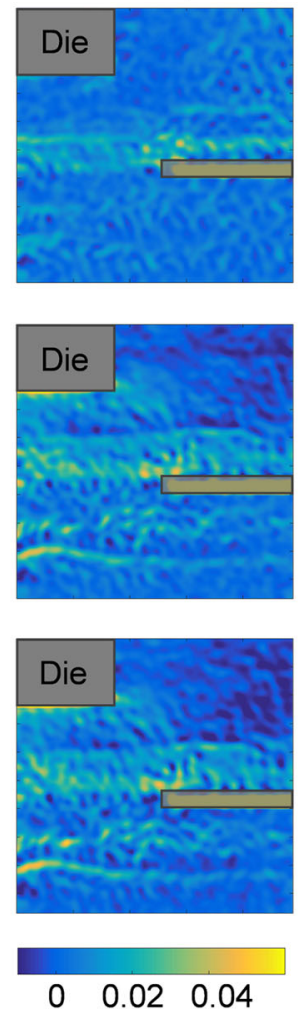

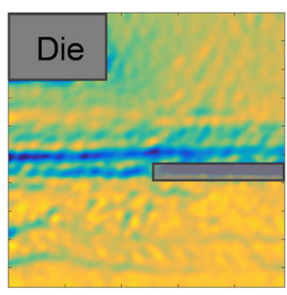

(e) Minimum principal
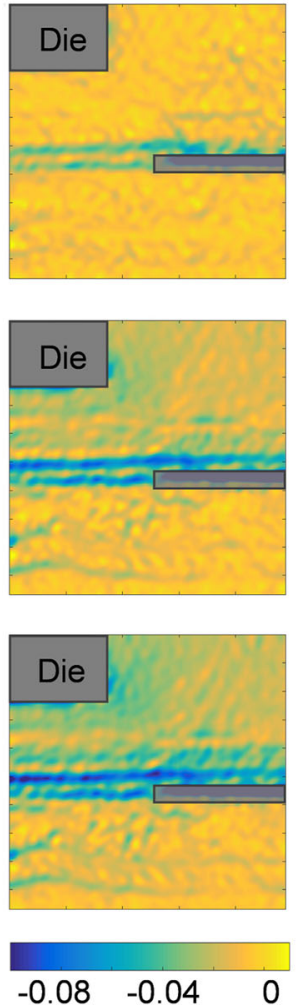

Fig. 13 Residual thermal strain distributions of FCPKG with UF-B relative to $150^{\circ} \mathrm{C}$ : normal strains in (a) $x$ direction and (b) $y$ direction, (c) shear strain, and (d) maximum and (e) minimum principal strains 
FCPKG, and the main mechanical and thermal material parameters are indicated in Appendix 2. The analysis is on thermal strain, during the process of temperature reduction from $150{ }^{\circ} \mathrm{C}$ (strain is 0 ) to $25^{\circ} \mathrm{C}$.

To compare with the simulation results, the residual strain distributions relative to the free contraction state at $25{ }^{\circ} \mathrm{C}$ of both underfills were measured using Eqs. (10)-(12), where the used CTE curves of underfills were plotted in Appendix 2. Then the residual principal strain distributions relative to the free contraction state at $25^{\circ} \mathrm{C}$ were calculated using Eq. (13). Since the CTEs of underfills, the buffer layer and the substrate are different and the emphasis is on underfills, only the residual principal strain distributions in the underfill area are compared with the simulation results.

Figure 15 illustrates the maximum residual principal strains at $25^{\circ} \mathrm{C}$ measured by experiments and simulations. For either UF-A and UF-B, the strain distribution trends from the experiment and the simulation are consistent. The maximum principal strain of UF-A with low $T g$ concentrates at the lower part of the die and the die corner, while that of UF-B with high $T g$ is greater near the die and at the buffer layer. The maximum principal strain of UF-A is higher than that of UF-B around the die.

Although the strain trends are the same, the strain values from experiments and simulations are a little bit different. The maximum principal strain of UF-A around the die has a maximum value of 0.06 from the experiment, whereas the maximum value is 0.04 from the simulation. The maximum value of the maximum principal strain of UF-B around the die from the experiment is 0.03 , also higher than the maximum value of 0.02 obtained by the simulation. It is presumed that the actual UF has low elasticity on the lower surface caused by filler sedimentation, but the UF with high elasticity is used in FEM. The other possible reason lies in the influence of the non-uniformity of the actual filler dispersion in the UF, whereas the UF is uniform in the FEM simulation.

A future work is to compare the proposed image processing technique with other methods such as the hole-drilling method and X-ray analysis in terms of residual strain measurement. Another future work is to improve the FEM model in numerical analysis in the light of experimental results.
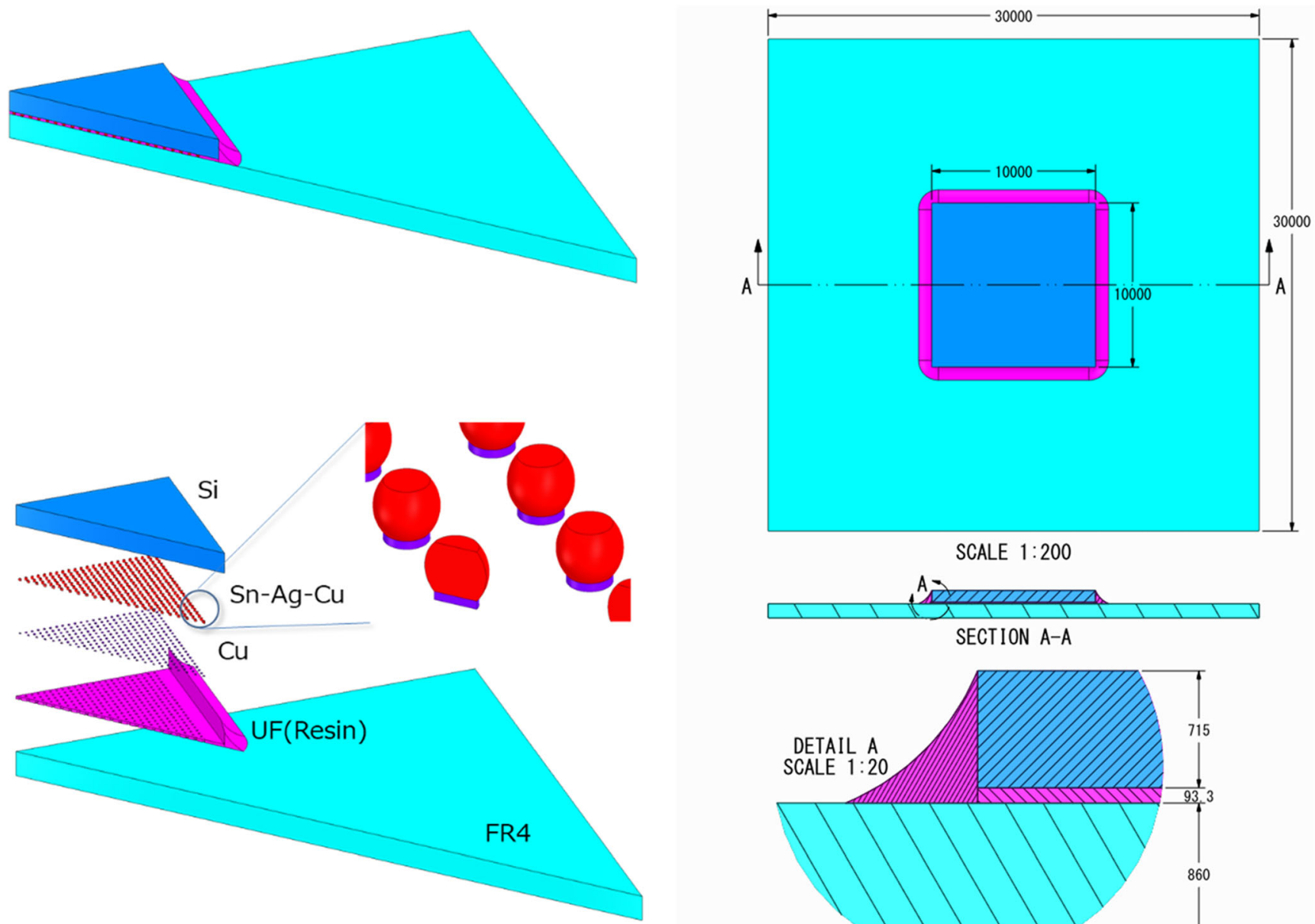

SCALE $1: 200$

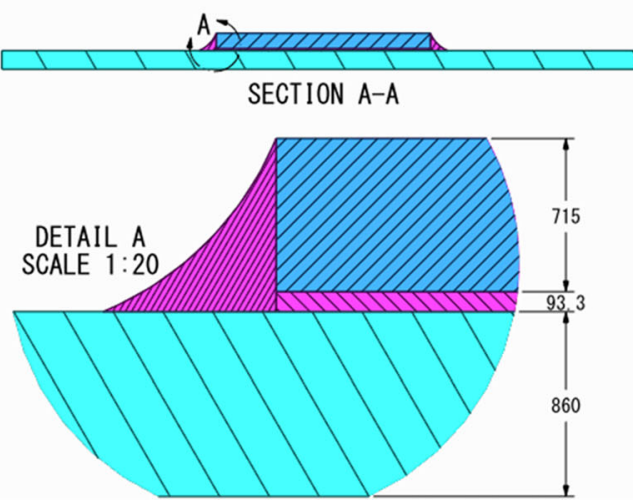

Fig. 14 Simulation model of FCPKG used in FEM for residual strain analysis 


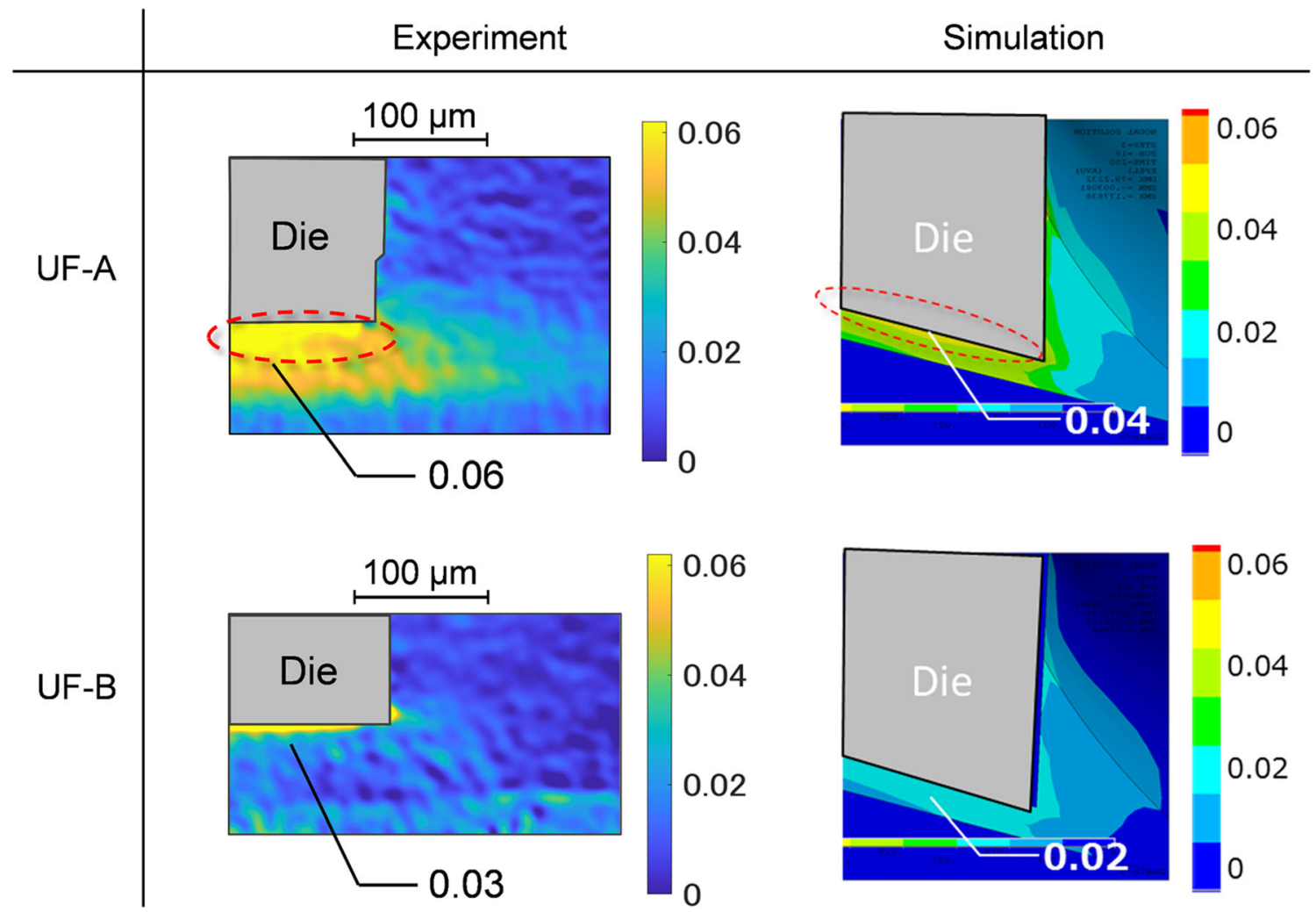

Fig. 15 Comparison of the maximum principal strains at $25^{\circ} \mathrm{C}$ relative to the free contraction state measured by experiments and simulations

Fig. 16 Thermal displacement distributions of underfills at $150{ }^{\circ} \mathrm{C}$ relative to $25^{\circ} \mathrm{C}$, where the displacement at the die corner is set as zero

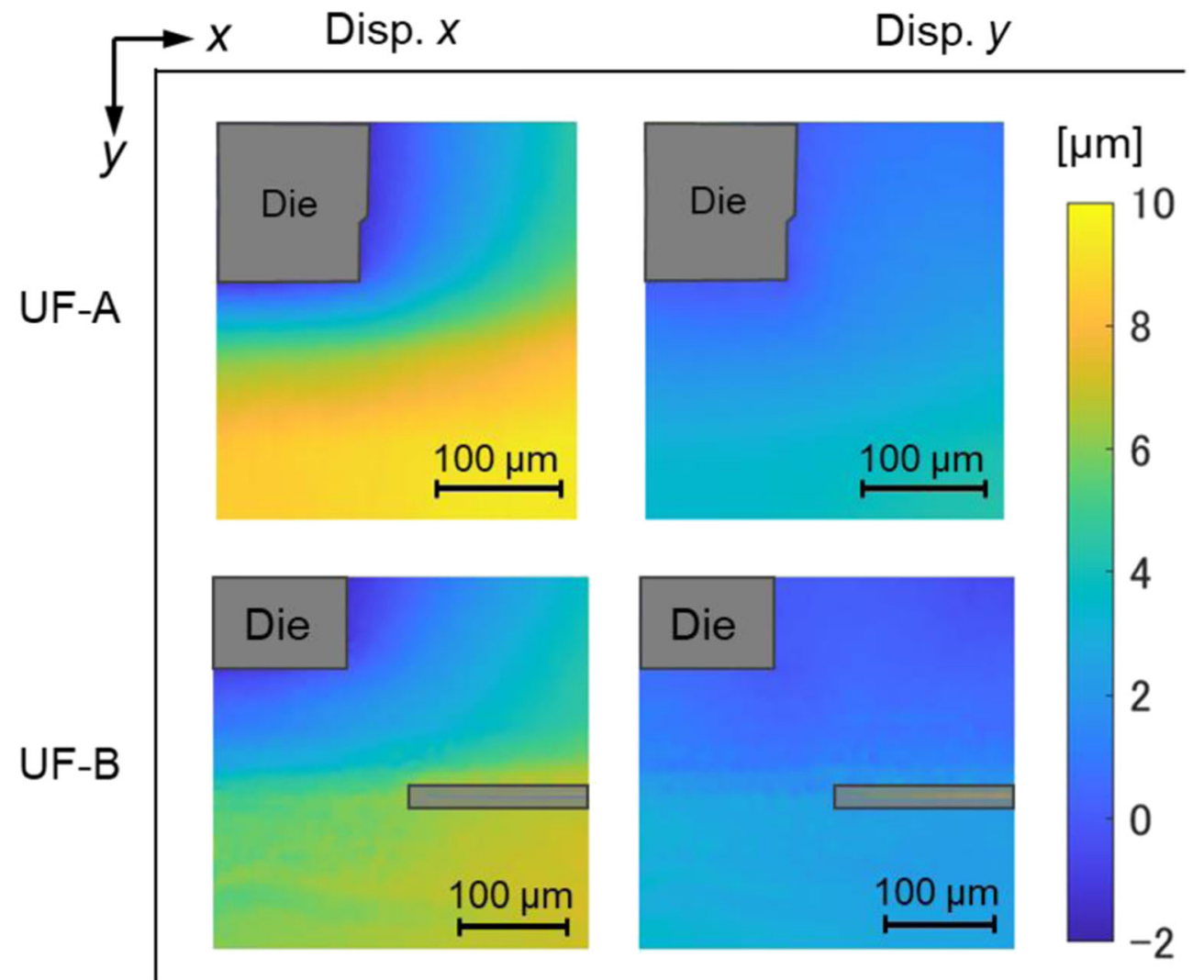




\section{Conclusions}

The inverse approach based on the sampling moiré method was proposed for measuring the internal thermal strain distributions nondestructively. The measurement principle of the residual strain at different temperatures relative to the specimen formation temperature or the free contraction state was developed. The strain measurement accuracy was verified from the numerical simulation. To investigate the residual thermal strain distributions measurement of UFs in FCPKGs which are most concerned, the clamping jig for nanoimprint lithography and the thermal chamber under the laser scanning microscope were designed. The normal, shear and principal internal strain distributions of two kinds of UFs at $125{ }^{\circ} \mathrm{C}$, $75^{\circ} \mathrm{C}$ and $25^{\circ} \mathrm{C}$ were measured relative to $150{ }^{\circ} \mathrm{C}$. The residual strains of UF-A with low $T g$ had greater absolute values around the die, especially at the die corner, whereas the strain localization of UF-B with high $T g$ mainly emerged at the buffer layer. The residual strain distributions trends from experiments are the same as that from FEM simulations. The proposed method is expected to be used for measuring the internal thermal strains in thermal cycle tests for predicting the life of UFs, finding the cause of crack formation, and optimizing the UF resin design.

\section{Appendix: Displacement and Parameters}

\section{Appendix 1: Thermal displacement distributions of underfills}

The thermal displacement distributions of two types of underfills at the specimen formation temperature $150{ }^{\circ} \mathrm{C}$ relative to the grid fabrication temperature $25^{\circ} \mathrm{C}$ calculated by the sampling moiré method are depicted in Fig. 16.

\section{Appendix 2: Material Parameters of FCPKGs}

The used mechanical and thermal material parameters of FCPKGs in FEM for simulation analysis are listed in Table 1.

Table 1 Material parameters of FCPKGs in FEM, where $E$ and $v$ are elastic modulus and poisson's ratio, respectively, and CTE represents the coefficient of thermal expansion

\begin{tabular}{llll}
\hline & $E(\mathrm{MPa})$ & $v$ & $\mathrm{CTE}(/ \mathrm{K})$ \\
\hline $\mathrm{Si}$ & $1.68 \times 10^{5}$ & 0.28 & $4.00 \times 10^{-6}$ \\
$\mathrm{Sn}-\mathrm{Ag}-\mathrm{Cu}$ & $76,087-109 \times T(\mathrm{~K})$ & 0.3 & $2.90 \times 10^{-5}$ \\
$\mathrm{Cu}$ & $1.17 \times 10^{5}$ & 0.34 & $1.72 \times 10^{-5}$ \\
$\mathrm{UF}-\mathrm{A}$ & Visco-elastic & 0.3 & Temp.dependent \\
$\mathrm{T}_{\mathrm{g}}(345 \mathrm{~K})$ & Visco-elastic & 0.3 & Temp.dependent \\
UF-B & Visco-elastic & 0.28 & Temp.dependent \\
$\mathrm{T}_{\mathrm{g}}(380 \mathrm{~K})$ & & & \\
FR4 & &
\end{tabular}

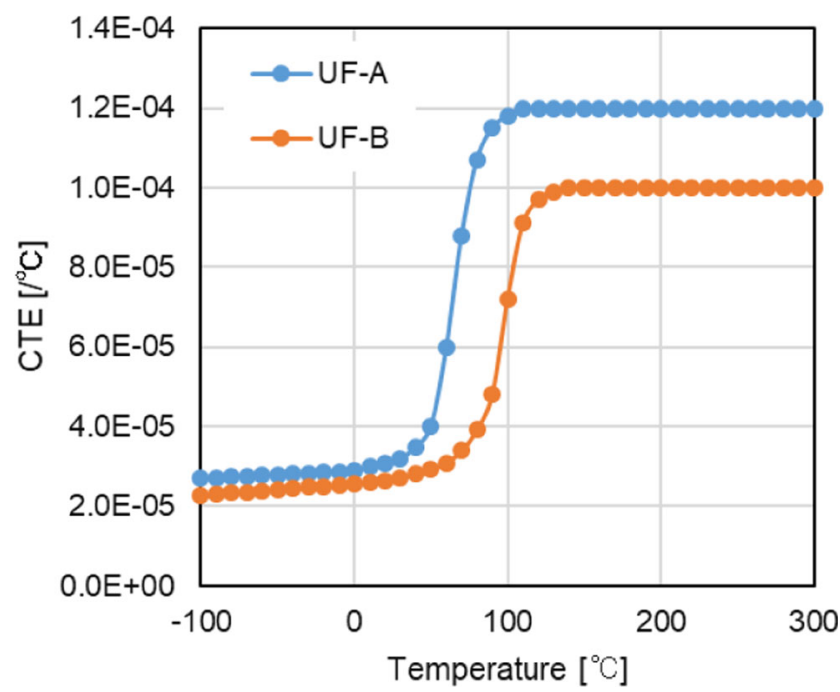

Fig. 17 Coefficient of the thermal expansion (CTE) curves of underfills

Besides, the variation curves of the coefficient of thermal expansion (CTE) of the used two types of underfills versus temperature are listed in Fig. 17.

Open Access This article is licensed under a Creative Commons Attribution 4.0 International License, which permits use, sharing, adaptation, distribution and reproduction in any medium or format, as long as you give appropriate credit to the original author(s) and the source, provide a link to the Creative Commons licence, and indicate if changes were made. The images or other third party material in this article are included in the article's Creative Commons licence, unless indicated otherwise in a credit line to the material. If material is not included in the article's Creative Commons licence and your intended use is not permitted by statutory regulation or exceeds the permitted use, you will need to obtain permission directly from the copyright holder. To view a copy of this licence, visit http://creativecommons.org/licenses/by/4.0/.

\section{References}

1. Chien C-H, Chen Y-C, Hsieh C-C, Chiou Y-T, Wu Y-D, Chen T-P (2004) Thermomechanical behavior of underfill/solder mask/substrate interface under thermal cycling. Exp Mech 44(2): 214-220

2. Ifju P, Han B (2010) Recent applications of moiré interferometry. Exp Mech 50(8):1129-1147

3. Patorski K (1993) Handbook of the moiré fringe technique. Elsevier Science,

4. Kehoe L, Guenebaut V, Kelly P Measurement of deformation and strain in flip chip on BGA (FC-BGA). In: Electronic Components and Technology Conference, 2004. Proceedings. 54th, 2004. IEEE, pp 120-127

5. Huang X, Liu Z, Xie H (2013) Recent progress in residual stress measurement techniques. Acta Mech Solida Sin 26(6):570-583

6. Wang Q, Xie H, Liu Z, Lou X, Wang J, Xu K, Zhang Z, Liao J, Gu C (2010) Residual stress assessment of interconnects by slot milling with FIB and geometric phase analysis. Opt Laser Eng 48(11): $1113-1118$

7. Jang W, Lee B-W, Kim D-W, Nah J-W, Paik K-W, Kwon D Evaluation of thermal shear strains in flip-chip package by electronic speckle pattern interferometry (ESPI). In: Electronic Materials 
and Packaging, 2001. EMAP 2001. Advances in, 2001. IEEE, pp 310-314

8. Kishimoto S, Egashira M, Shinya N (1993) Microcreep deformation measurements by a moiré method using electron beam lithography and electron beam scan. Opt Eng 32(3):522-526

9. Han B, Post D, Ifju P (2001) Moiré interferometry for engineering mechanics: current practices and future developments. J Strain Analy Eng Design 36(1):101-117

10. Ri S, Fujigaki M, Morimoto Y (2010) Sampling moiré method for accurate small deformation distribution measurement. Exp Mech 50(4):501-508

11. Lei ZK, Wang ZL Vibration testing parameters measured by sampling moire method. In: Applied Mechanics and Materials, 2012. Trans Tech Publ, pp 1975-1980

12. Zhang Q, Xie H, Liu Z, Shi WJO, Engineering Li (2018) Sampling moiré method and its application to determine modulus of thermal barrier coatings under scanning electron microscope 107:315-324

13. International Journal of Microcircuits and Electronic PackagingJournal of Thermal StressesMiller MR, Mohammed I, Ho PS (2001) Quantitative strain analysis of flip-chip electronic packages using phase-shifting moiré interferometry. Opt Laser Eng 36(2):127-139

14. Wang Q, Kishimoto S (2012) Simultaneous analysis of residual stress and stress intensity factor in a resist after UV-nanoimprint lithography based on electron moiré fringes. J Micromech Microeng 22(10): 105021

15. Li Y, Xie H, Wang Q, Zhou M, Xu M, Luo Q, Gu C (2016) The residual strain measurement of thin conductive metal wire after electrical failure with SEM moiré. Acta Mech Solida Sin 29(4): 371-378

16. Han B, Guo Y (1995) Thermal deformation analysis of various electronic packaging products by moiré and microscopic moiré interferometry. J Electron Packag 117(3):185-191

17. Cho S, Han B (2001) Effect of Underfill on Flip-Chip solder bumps: an experimental study by microscopic moiréinterferometry. Int J Microcirc Electron Packag 24(3):217239
18. Han B (2003) Thermal stresses in microelectronics subassemblies: quantitative characterization using photomechanics methods. J Therm Stresses 26(6):583-613

19. Morita Y, Arakawa K, Todo M, Kaneto M (2006) Experimental study on the thermo-mechanical effects of underfill and low-CTE substrate in a flip-chip device. Microelectron Reliab 46(5-6):923929

20. Post D, Han B (2008) Moiré interferometry. In: Springer Handbook of Experimental Solid Mechanics. Springer, pp 627-654

21. Wang Q, Ri S, Tsuda H, Koyama M, Tsuzaki K (2017) Twodimensional Moire phase analysis for accurate strain distribution measurement and application in crack prediction. Opt Express 25(12):13465-13480

22. Wang Q, Ri S, Tsuda H, Koyama M (2018) Optical full-field strain measurement method from wrapped sampling Moiré phase to minimize the influence of defects and its applications. Opt Laser Eng 110:155-162

23. Omens JH, Fung Y CJCr (1990) Residual strain in rat left ventricle. $66(1): 37-45$

24. Ri S, Agarwal N, Wang Q, Kemao Q (2018) Comparative study of sampling moiré and windowed Fourier transform techniques for demodulation of a single-fringe pattern. Appl Opt 57(36):1040210411

25. Hirasawa T, Taniguchi J, Ohtaguchi M, Sakai N (2007) Photocurable resins and the evaluation methods for UV-Nanoimprint lithography. IEEJ Trans Electron Inform Syst 127:174-178

26. Wang Q, Kishimoto S, Tanaka Y, Kagawa Y (2013) Micro/ submicro grating fabrication on metals for deformation measurement based on ultraviolet nanoimprint lithography. Opt Laser Eng 51(7):944-948

27. Bi H, Jang C, Han B (2010) Nano-pattern recognition and correlation technique for sub-nanometer in-plane displacement measurement. Exp Mech 50 (8):1169-1181

Publisher's Note Springer Nature remains neutral with regard to jurisdictional claims in published maps and institutional affiliations. 